\title{
Neuronal Morphology Generates High-Frequency Firing Resonance
}

\author{
- DSrdjan Ostojic, ${ }^{1}$ Germán Szapiro, ${ }^{2,3,4}$ Eric Schwartz, ${ }^{2,3,4}$ Boris Barbour, ${ }^{2,3,4}$ Nicolas Brunel, ${ }^{6,7}$ and Vincent Hakim ${ }^{5}$ \\ ${ }^{1}$ Laboratoire de Neurosciences Cognitives, Institut National de la Santé et de la Recherche Médicale (INSERM) Unité 960, ${ }^{2}$ Ecole Normale Supérieure, \\ Institute de Biologie (IBENS), ${ }^{3}$ Centre National de la Recherche Scientifique (CNRS) Unité Mixte de Recherche (UMR) 8197, and ${ }^{4}$ INSERM Unité 1024, \\ Paris, F-75005 France, ${ }^{5}$ Laboratoire de Physique Statistique, CNRS UMR 8550, Ecole Normale Supérieure, F-75231 Paris, France, and Departments of \\ ${ }^{6}$ Statistics and ${ }^{7}$ Neurobiology, University of Chicago, Chicago, Illinois 60637
}

The attenuation of neuronal voltage responses to high-frequency current inputs by the membrane capacitance is believed to limit single-cell bandwidth. However, neuronal populations subject to stochastic fluctuations can follow inputs beyond this limit. We investigated this apparent paradox theoretically and experimentally using Purkinje cells in the cerebellum, a motor structure that benefits from rapid information transfer. We analyzed the modulation of firing in response to the somatic injection of sinusoidal currents. Computational modeling suggested that, instead of decreasing with frequency, modulation amplitude can increase up to high frequencies because of cellular morphology. Electrophysiological measurements in adult rat slices confirmed this prediction and displayed a marked resonance at $200 \mathrm{~Hz}$. We elucidated the underlying mechanism, showing that the two-compartment morphology of the Purkinje cell, interacting with a simple spiking mechanism and dendritic fluctuations, is sufficient to create high-frequency signal amplification. This mechanism, which we term morphology-induced resonance, is selective for somatic inputs, which in the Purkinje cell are exclusively inhibitory. The resonance sensitizes Purkinje cells in the frequency range of population oscillations observed in vivo.

Key words: computational modelling; information bandwidth; neural dynamics; Purkinje cell

\section{Introduction}

Single-neuron properties constrain higher-level computations but have presumably also evolved to serve the computational needs of the brain in terms of speed, a fundamental requirement of information processing by nervous systems. The neuronal membrane time constant appears to impose a stringent temporal constraint on the single-cell response to time-varying inputs. However, it is known that higher cognitive tasks, such as discriminating an animal from an object, can be performed in $<200 \mathrm{~ms}$ by human subjects (Rousselet et al., 2002), and information about object category is available as early as $100 \mathrm{~ms}$ after a stimulus presentation (Liu et al., 2009). This conundrum has led several groups to investigate whether and how the limit imposed by the membrane time constant can be bypassed. It has been shown both theoretically (Tsodyks and Sejnowski, 1995; van Vreeswijk and Sompolinsky, 1998; Brunel et al., 2001; Lindner

\footnotetext{
Received Sept. 19, 2014; revised March 12, 2015; accepted March 20, 2015.

Author contributions: S.O., B.B., N.B., and V.H. designed research;S.O., G.S., E.S., B.B., N.B., and V.H. performed research;S.O. and B.B. analyzed data; S.O., B.B., N.B., and V.H. wrote the paper.

This work was supported by National Agency for Research Grant DALTPAC (B.B., N.B., and V.H.). This work has received support under the program "Investissements d'Avenir" launched by the French Government and implemented by the National Agency for Research, with the references ANR-10-LABX-0087 IEC, ANR-10-LABX-54 MEMO LIFE, and ANR-11-IDEX-0001-02 PSL* Research University.

The authors declare no competing financial interests.

Correspondence should be addressed to Srdjan 0stojic, Laboratoire de Neurosciences Cognitives, Ecole Normale Supérieure, 29 rue d'Ulm, F-75005 Paris, France. E-mail: srdjan.ostoji@@ens.fr.

E. Schwartz's present address: Institut National de la Santé et de la Recherche Médicale, Institut du Fer à Moulin, F-75005 Paris, France.

DOI:10.1523/JNEUROSCI.3924-14.2015

Copyright $\odot 2015$ the authors $\quad 0270-6474 / 15 / 357056-13 \$ 15.00 / 0$
}

and Schimansky-Geier, 2001) and experimentally (Köndgen et al., 2008; Boucsein et al., 2009; Tchumatchenko et al., 2011; Testa-Silva et al., 2014) that a population of neurons can respond on the millisecond timescale to a changing stimulus presented on a background of fluctuating inputs. However, the underlying mechanism leading to such fast timescales is still the subject of debate.

The temporal response of a neuron can be quantified by measuring the firing-rate modulation induced by oscillating inputs of different frequencies superposed on a noisy background input mimicking in vivo conditions (Brunel and Hakim, 1999; Brunel et al., 2001; Lindner and Schimansky-Geier, 2001). This firing-rate response function allows one to describe the cell output spike response to arbitrary temporal inputs (Ostojic et al., 2009; Ostojic and Brunel, 2011). Previous experimental work has measured the firing-rate modulation of neocortical neurons (Köndgen et al., 2008; Boucsein et al., 2009; Tchumatchenko et al., 2011; Broicher et al., 2012) and has found a flat frequency response that is only attenuated above frequencies of a few hundred Hertz, a bandwidth that would allow a population of neocortical neurons to respond within $1 \mathrm{~ms}$ to somatic inputs. The mechanisms underlying such high cutoff frequencies are still unclear, with very recent work indicating a role for dendritic impedance (Eyal et al., 2014).

The firing-rate response has also served to characterize the frequency preference of a neuron and the possible existence of a resonant frequency (Brunel et al., 2003; Richardson et al., 2003), which, when present, is generally observed in the range of a few 
Hertz to a few tens of Hertz. The mechanisms of such frequency preference established so far rely on the expression of particular voltage-gated ionic conductances, the activation of which can create subthreshold (Hutcheon and Yarom, 2000; Hu et al., 2002, 2009; Richardson et al., 2003; Narayanan and Johnston, 2008; Zemankovics et al., 2010) or suprathreshold resonance, with the frequency preference in the latter case resulting from spikefrequency adaptation (Fuhrmann et al., 2002).

In the present work, we explore possible mechanisms that might extend neuronal bandwidth beyond the capacitive limit. We focus on the Purkinje cell, the sole output neuron of the cerebellar cortex. The cerebellum is involved in the learning and control of coordinated movements that require rapid and precise timing abilities. We measured the firing-rate modulation of Purkinje cells and found that little attenuation occurs below very high frequencies of $\sim 1 \mathrm{kHz}$ and, furthermore, that somatic inputs are actually amplified up to frequencies $\sim 200 \mathrm{~Hz}$. This response spectrum allows Purkinje cells to respond extremely rapidly and to participate optimally in the fast cerebellar oscillation (de Solages et al., 2008). Moreover, this fast response ability does not depend on the expression of a specific ionic conductance. Electrophysiological experiments and computational modeling lead us to identify a new mechanism of amplification that relies on the Purkinje cell anatomy with its large dendritic arbor. Morphology-induced resonance is optimal when rapidly varying inputs are targeted specifically to the soma in the presence of dendritic noise, which can arise from background synaptic input.

\section{Materials and Methods}

\section{Electrophysiology}

Experimental protocols conformed to French and European animal welfare guidelines. Preparation of sagittal cerebellar slices from adult male Wistar rats essentially followed the methods of Isope and Barbour (2002). Minor differences were as follows: (1) animals were not ventilated and a tracheal catheter was therefore unnecessary (the establishment of the transcardiac perfusion was sufficiently rapid to preclude ischemic damage); and (2) kynurenate was not added to the slicing solution. The animals were housed with a light cycle such that the lights were on 7:00 P.M. and 7:00 A.M.; slices were generally prepared in the morning.

Recordings were also performed according to the methods of Isope and Barbour (2002), with some differences. The pipette solution was potassium based and was usually either a fairly classical potassium-gluconate recipe or a more elaborate one designed to improve energy supply within the cell. We do not believe that these changes strongly influenced the high-frequency firing properties of the Purkinje cells. The classical intracellular solution contained the following (in mM): $150 \mathrm{~K}$-gluconate, $4 \mathrm{NaCl}, 10 \mathrm{HEPES}, 1 \mathrm{MgCl}_{2}, 0.005 \mathrm{~K}_{3} \mathrm{EGTA}, 6 \mathrm{Mg}_{2} \mathrm{ATP}$, and $0.4 \mathrm{NaGTP}$, adjusted to $\mathrm{pH} 7.3$ with $\mathrm{KOH}(290-300 \mathrm{mOsm})$. We also used the following pipette solution: 3.5 ATP-Mg salt, 0.4 GTP-Na salt, 0.5 L-(-)malic acid, 0.008 oxaloacetic acid, $0.18 \alpha$-ketoglutaric acid, 0.2 pyridoxal $5^{\prime}$-phosphate hydrate, 5 L-alanine, 0.15 pyruvic acid, 15 L-glutamine, 4 L-asparagine, 1 reduced L-glutathione, $0.5 \mathrm{NAD}^{+}, 5 \mathrm{~K}_{2}$-phosphocreatine, $148 \mathrm{~K}$-gluconate, $10 \mathrm{HEPES}, 0.5$ EGTA, $4 \mathrm{KCl}, 2.5 \mathrm{~K}_{2}$ phosphate, $0.15-0.2 \mathrm{CaCl}_{2}$, and $1.5 \mathrm{MgCl}_{2}$. The solution was adjusted to $\mathrm{pH} 7.3$ with $\mathrm{KOH}$ and to $290-300 \mathrm{mOsm}$. The same recipe was also used with an additional $0.05 \mathrm{~mm} \mathrm{CaCl}_{2}$ or $3 \mathrm{~mm} \mathrm{Na}$-acetate. A potassiummethane sulfonate solution was also used, containing the following: 140 mм $\mathrm{KMeSO}_{4}, 6 \mathrm{~mm} \mathrm{NaCl}, 2 \mathrm{~mm} \mathrm{MgCl}$, 10 mм HEPES, 4 mм MgATP, 0.5 mM Na $\mathrm{Na}_{3} \mathrm{GT}, 10 \mathrm{~mm}$ phosphocreatine, and $0.3 \mathrm{~mm}$ EGTA, adjusted to $\mathrm{pH}$ 7.4 with $1 \mathrm{M} \mathrm{KOH}$ and to $300 \mathrm{mOsm}$. The extracellular solution from Isope and Barbour (2002) was on occasion supplemented with $0.5 \mathrm{~mm}$ glutamine and/or $0.25 \mathrm{~mm}$ pyruvate. Membrane potentials have not been corrected for the $\sim 8 \mathrm{mV}$ liquid junction potential at the recording temperature of $32^{\circ} \mathrm{C}$. We usually allowed Purkinje cells to fire spontaneously; occasionally, positive or negative bias currents of 100-200 pA were injected.

Noise was generated in real time by differential amplification $(10,000 \times)$ of the thermal noise in a pair of $10 \mathrm{M} \Omega$ resistors. Filtering limited the bandwidth to $\sim 3 \mathrm{kHz}$. Within this window, the noise closely approximated Gaussian white noise. The noise was added to the current command, giving an SD of tens of picoamperes; the precise values are given in text.

A majority of Purkinje cells entered a "trimodal" firing pattern (Womack and Khodakhah, 2002) after a variable period of tonic firing after entry into whole-cell mode. These cells would alternate between tonic firing, bursts with underlying calcium spikes, and silence. The onset of bursts would be signaled by increasing frequency, the appearance of spike doublets, and then short bursts. Only periods of tonic firing were analyzed, and this was automated by excluding sweeps ( $5 \mathrm{~s}$ long) during which the mean frequency exceeded 100 $\mathrm{Hz}$ or the coefficient of variation (CV) exceeded 0.7.

For an artificial firing mechanism, we constructed an electronic circuit implementing a hard threshold on the recorded membrane potential; on each threshold crossing, a reset current pulse was added to the current command (see diagram in Fig. 8A). The threshold used a fast comparator (AD790) that triggered the reset pulse (LM555), whose duration and amplitude (signal routed via a DG419 analog switch) were controlled by potentiometers, as was the voltage threshold of the comparator. The pulses constituted the output "spike" train. The circuit diagrams are available from Boris Barbour on request. All electronic components were purchased through Farnell.

These recordings used the "elaborate" pipette solution described above, without NAD and with the addition of $5 \mathrm{~mm}$ each tetraethylammonium (TEA)-Cl and lidocaine $\mathrm{N}$-ethyl bromide (QX 314); the solution osmolarity was returned to $300 \mathrm{mOsm}$ by dilution with distilled water. The extracellular calcium concentration was 1 or $2 \mathrm{~mm}$.

Firing-rate modulation in response to oscillatory input currents We examined the modulation of the instantaneous firing rate $\nu(t)$ by a sinusoidally modulated applied current at frequency $f$ :

$$
I_{\text {inp }}=I_{1} \sin (2 \pi f t) .
$$

The firing rate $\nu(t)$ is computed by averaging the instantaneous firing rate of the neuron over different trials.

If the amplitude $I_{1}$ of the current modulation is small, the firing-rate modulation around the baseline firing rate is expected to be sinusoidal and proportional to $I_{1}$ :

$$
v(t)=v_{0}+I_{1} A(f) \sin (2 \pi f+\phi(f)) .
$$

Here $v_{0}$ is the baseline firing rate corresponding to the spontaneous firing of the Purkinje cell. The modulation of the firing rate at frequency $f$ is thus fully specified by its amplitude $A(f)$ and phase lag $\phi(f)$ of the sinusoidal component. Equivalently, one can use the complex firing-rate response defined by $R(f)=A(f) e^{i \phi(f)}$

\section{Single-compartment models of the Purkinje cell}

In a single-compartment model of a neuron, the membrane potential is considered to be uniform over the whole cell. Its dynamics are given by the following:

$$
C \frac{d V}{d t}=-g_{l} V+I_{\mathrm{act}}+I_{\mathrm{inp}}+I_{\mathrm{syn}}
$$

where the membrane potential $V$ is determined with respect to the resting potential of the cell. The input currents on the right side correspond to active membrane currents $\left(I_{\text {act }}\right)$, external input currents $\left(I_{\text {inp }}\right)$, and background synaptic inputs $\left(I_{\text {syn }}\right)$. The membrane capacitance $C$ and conductance $g_{l}$ are proportional to the area $A$ of the compartment: $C=A c_{m}$ and $g_{l}=A g_{m}$, where $c_{m}$ and $g_{m}$ are the specific capacitance and conductance of the membrane. The specific time constant is independent of the area of the compartment and given by $\tau_{m}=c_{m} / g_{m}$. For the Purkinje cells in vitro, $\tau_{m}$ is close to $50 \mathrm{~ms}$ (Rapp et al., 1994; Roth and Häusser, 2001).

Khaliq-Gouwens-Raman model. The spontaneous firing of the Purkinje cell has been shown to be attributable to a pacemaking mechanism 
based on an interplay between potassium channels and resurgent sodium channels (Raman and Bean, 2001; Khaliq et al., 2003; Akemann and Knöpfel, 2006). To examine the influence of this pacemaking mechanism on the modulation of the firing rate, we studied the behavior of the detailed conductance-based model developed by Khaliq, Gouwens, and Raman (KGR) for dissociated Purkinje cells (Khaliq et al., 2003). The model consisted of a resurgent sodium current, three potassium currents (TEA-sensitive, low TEA-sensitive, and slow K), a P-type calcium current, a B-type calcium-dependent potassium current, and a hyperpolarization-activated $I_{h}$ current. The values of all activation parameters and maximal conductances were taken from the study by Khaliq et al. (2003). The background synaptic activity was modeled by including 200 conductance-based AMPA synapses with a rise time of 0.2 $\mathrm{ms}$ and a decay time of $2 \mathrm{~ms}$ (for computational efficiency, inhibitory inputs were included as tonic hyperpolarizing currents). The presynaptic firing rate and the maximal synaptic conductances were chosen so as to produce the desired rate $(30-50 \mathrm{~Hz})$ and variability $(\mathrm{CV}$ of $\sim 0.6)$ in the firing of the Purkinje cell. This model was studied using NEURON 6.0.

Exponential integrate-and-fire model. The exponential integrate-andfire (EIF) model (Fourcaud-Trocmé et al., 2003) is a nonlinear integrateand-fire model that provides a simplified, exponential spike-generating current:

$$
I_{\text {act }}(V)=g_{l} \Delta_{T} \exp \left(\frac{V-V_{T}}{\Delta_{T}}\right)
$$

Once the membrane potential crosses the threshold $V_{T}$, it diverges to infinity in finite time. This divergence represents the firing of an action potential (AP). After the divergence, the membrane potential is reset instantaneously to a value $V_{r}$ after a refractory period $\tau_{r p}$. The parameter $\Delta_{T}$ represents the voltage range below threshold over which the exponential current is activated and therefore quantifies the sharpness of the AP initiation. For the Purkinje cell, $\Delta_{T} \sim 0.75 \mathrm{mV}$ (Rieubland et al., 2008), whereas for pyramidal neurons, $\Delta_{T} \sim 1.2 \mathrm{mV}$ (Badel et al., 2008), i.e., the spike initiation is sharper in Purkinje cells than in pyramidal neurons. For the other parameters, we used $V_{T}=15 \mathrm{mV}, V_{r}=5 \mathrm{mV}$, and $\tau_{r p}=0.1$ $\mathrm{ms}$. In this model, spontaneous firing was induced by injecting a constant tonic current, and the cumulative effect of many co-occurring synaptic inputs was approximated by a Gaussian white noise, whose mean and SD were chosen so as to produce the desired firing rate $(30-50 \mathrm{~Hz})$ and firing variability ( $\mathrm{CV}$ of $\sim 0.6)$.

\section{Multicompartment model of the Purkinje cell}

Morphology. We used a freely available, multicompartment model based on a detailed morphological reconstruction of a Purkinje cell from a guinea pig (Shelton, 1985). The model contained 1088 compartments (soma, 1 compartment; smooth dendrites, 85 compartments; spiny dendrites, 1002 compartments). The specific capacitance was set to $0.8 \mu \mathrm{F} /$ $\mathrm{cm}^{2}$ in the soma and smooth dendrites. The dendritic spines were not described as independent compartments, instead, to incorporate them in the model, the specific capacitance of spiny dendrites was set to 1.5 $\mu \mathrm{F} / \mathrm{cm}^{2}$. The internal axial resistivity was set to $250 \Omega / \mathrm{cm}$, and the specific resistivity of soma and dendrites was $5 \mathrm{k} \Omega / \mathrm{cm}^{2}$, resulting in an input resistance of $20 \mathrm{M} \Omega$ (Rapp et al., 1994).

Ion channels. The channels corresponding to the KGR pacemaker were inserted in the soma of the multicompartment model. Because that pacemaker model was developed initially for dissociated somata of Purkinje cells, once introduced in the multicompartment morphology, the maximal conductances of the channels needed to be adapted. We scaled these values by a factor of 8 with respect to the original model. In the dendrites, we inserted only the $I_{h}$ channel at a maximal conductance of $2.5 \mathrm{mS} / \mathrm{cm}^{2}$.

Excitatory synapses were inserted only in the spiny dendrites, with a uniform density. The presynaptic firing rate and the maximal synaptic conductances were chosen so as to produce the desired rate $(30-50 \mathrm{~Hz})$ and variability ( $\mathrm{CV}$ of $\sim 0.6)$ in the firing of the Purkinje cell. The precise values of these parameters did not affect the results concerning the firingrate response function. The multicompartment model was studied using NEURON 6.0 .

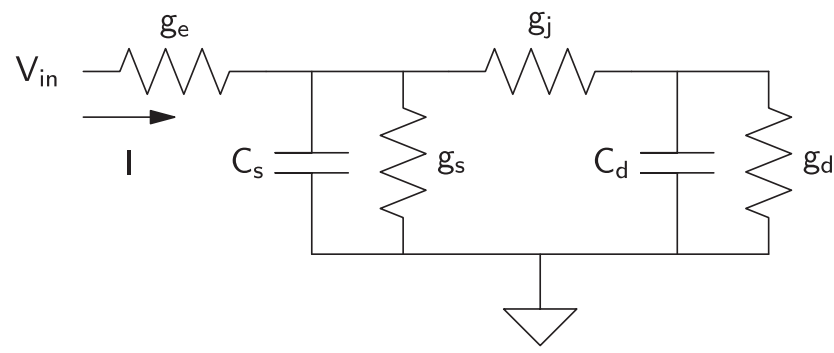

Figure 1. Electrical circuit equivalent to the two-compartment model: $g_{e}$ is the conductance of the electrode, $g_{s}$ and $C_{s}$ are the conductance and capacitance of the somatic compartment, $g_{d}$ and $C_{d}$ are the conductance and capacitance of the dendritic compartment, and $g_{j}$ is the junctional conductance between the two compartments.

Two-compartment model of the Purkinje cell

Two-compartment models have been useful to understand the influence of neuronal morphology in a simplified setting (Pinsky and Rinzel, 1994). The two-compartment model of the Purkinje cell (Llano et al., 1991; Fig. 1) consists of a compartment containing mechanisms for generating APs and another compartment with only subthreshold conductances. For convenience, we refer to them as the "somatic" and the "dendritic" compartments (Roth and Häusser, 2001). The dynamics of the membrane potentials $V_{s}$ and $V_{d}$ in the two compartments are given by the following:

$$
\begin{gathered}
C_{s} \frac{d V_{s}}{d t}=-g_{s} V_{s}+g_{j}\left(V_{d}-V_{s}\right)+I_{\mathrm{act}, s}+I_{\mathrm{inp}, s}+I_{\mathrm{syn}, s}, \\
C_{d} \frac{d V_{d}}{d t}=-g_{d} V_{d}+g_{j}\left(V_{s}-V_{d}\right)+I_{\mathrm{inp}, d}+I_{\mathrm{syn}, d} .
\end{gathered}
$$

The input currents on the right side correspond to active currents $I_{\text {act, }, s}$, external input currents $\left(I_{\mathrm{inp}, s}, I_{\mathrm{inp}, d}\right)$, and background synaptic inputs $\left(I_{\text {syn }, s}, I_{\text {syn }, d}\right)$.

In the two-compartment model, spike initiation was again implemented using the EIF mechanism (Fourcaud-Trocmé et al., 2003), as described for the one-compartment model above but with a different prefactor: $I_{\text {act }, s}=\left(g_{s}+g_{j}\right) \Delta_{T} \exp \left(\frac{V-V_{T}}{\Delta_{T}}\right)$. In the absence of other hyperpolarizing currents, this somatic reset is not sufficient to significantly hyperpolarize the dendritic compartment. To reproduce approximately the post-spike hyperpolarization measured experimentally in the dendrites (Häusser et al., 2001), after an AP, the membrane potential in the dendritic compartment was decreased by $\beta=0.5 \mathrm{mV}$.

The conductance $g_{j}$ represents the junctional conductance between the two compartments. The membrane capacitances $C_{s}$ and $C_{d}$ and conductances $g_{s}$ and $g_{d}$ are proportional to the areas $A_{s}$ and $A_{d}$ of the compartments: $C_{s}=A_{s} c_{m}, C_{d}=A_{d} c_{m}, g_{s}=A_{s} g_{m}$, and $g_{d}=A_{d} g_{m}$, where $c_{m}$ and $g_{m}$ are the specific capacitance and conductance of the membrane. Here we assumed that the specific membrane conductance $g_{m}$ is identical in the two compartments and that $c_{m}=1 \mu \mathrm{F} / \mathrm{cm}^{2}$.

It is useful to rewrite the Equations 5 for $V_{s}$ and $V_{d}$ after dividing them by the total membrane conductance of each compartment (respectively, by $g_{s}+g_{j}$ and $\left.g_{d}+g_{j}\right)$ :

$$
\tau_{s} \frac{d V_{s}}{d t}=-V_{s}+g_{j, s} V_{d}+\Delta_{T} \exp \left(\frac{V-V_{T}}{\Delta_{T}}\right)+\mu_{\text {inp }, s}+\mu_{\text {syn }, s},
$$

$$
\tau_{d} \frac{d V_{d}}{d t}=-V_{d}+g_{j, d} V_{s}+\mu_{\mathrm{inp}, d}+\mu_{\mathrm{syn}, d}
$$

where

$$
\tau_{s}=\frac{C_{s}}{g_{s}+g_{j}} \quad \tau_{d}=\frac{C_{d}}{g_{d}+g_{j}},
$$




$$
\begin{gathered}
g_{j, s}=\frac{g_{j}}{g_{s}+g_{j}} \quad g_{j, d}=\frac{g_{j}}{g_{s}+g_{j}}, \\
\mu_{\text {inp }, s}=\frac{I_{\text {inp }, s}}{g_{s}+g_{j}} \quad \mu_{\text {inp }, d}=\frac{I_{\text {inp }, d}}{g_{d}+g_{j}}, \\
\mu_{\text {syn }, s}=\frac{I_{\text {syn }, s}}{g_{s}+g_{j}} \quad \mu_{\text {syn }, d}=\frac{I_{\text {syn }, d}}{g_{d}+g_{j}} .
\end{gathered}
$$

The free parameters in the two-compartment model are the areas $A_{s}$ and $A_{d}$ of the compartments, the junctional conductance between the two compartments and the specific membrane resistance $g_{m}$. We determined the values of these parameters from fits to experimentally measured somatic current transients elicited by steps of holding voltage under voltage clamp (see Fig. 4C). This procedure introduces an additional parameter, the conductance $g_{e}$ of the electrode. In the two-compartment model, the transient current is a sum of two exponentials:

$$
I_{s}(t)=I_{1} e^{-\frac{t}{\tau_{1}}}+I_{2} e^{-\frac{t}{\tau_{2}}}+I(V) .
$$

The time constants are given by the following:

$$
\begin{aligned}
\frac{1}{\tau_{1}}=\frac{g_{e}+g_{s}+g_{j}}{2 C_{s}}+ & \frac{g_{j}+g_{d}}{2 C_{d}} \\
& +\sqrt{\frac{1}{4}\left(\frac{g_{e}+g_{s}+g_{j}}{C_{s}}-\frac{g_{j}+g_{d}}{C_{d}}\right)^{2}+\frac{g_{j}^{2}}{C_{s} C_{d}}},
\end{aligned}
$$

$\frac{1}{\tau_{2}}=\frac{g_{e}+g_{s}+g_{j}}{2 C_{s}}+\frac{g_{j}+g_{d}}{2 C_{d}}-$

$$
\sqrt{\frac{1}{4}\left(\frac{g_{e}+g_{s}+g_{j}}{C_{s}}-\frac{g_{j}+g_{d}}{C_{d}}\right)^{2}+\frac{g_{j}^{2}}{C_{s} C_{d}}},
$$

where $C_{s}=A_{s} c_{m}, C_{d}=A_{d} c_{m}, g_{s}=A_{s} g_{m}, g_{d}=A_{d} g_{m}$, and $c_{m}=1 \mu \mathrm{F} / \mathrm{cm}^{2}$.

The coefficients $I_{1}$ and $I_{2}$ can be expressed as functions of the parameters using the initial conditions.

Average parameter values obtained for $n=5$ cells from two animals were $A_{s}=2000 \pm 500 \mu \mathrm{m}^{2}, A_{d}=150,000 \pm 1500 \mu \mathrm{m}^{2}, g_{j}=0.17 \pm 0.04$ $\mu \mathrm{S}$, and $g_{m}=0.05 \pm 0.01 \mathrm{~S} / \mathrm{m}^{-2}$, leading to $C_{s}=20 \mathrm{pF}, C_{d}=1500 \mathrm{pF}$, $g_{s}=0.1 \mathrm{nS}$, and $g_{d}=7.5 \mathrm{nS}$. These were the default parameter values used in the two-compartment model (see Fig. 5).

For the parameter values determined from experiments, $g_{j} \gg g_{s}$ and $g_{j}$ $\gg g_{d}$, so that $\tau_{s} \sim c_{m} A_{s} / g_{j}, \tau_{d} \sim c_{m} A_{d} / g_{j}$. Because the area $A_{d}$ of the dendritic compartment is $>50$ times larger than the area $A_{s}$ of the somatic compartment, the effective timescales $\tau_{s}$ and $\tau_{d}$ are separated widely, with $\tau_{s}=0.11 \mathrm{~ms}$ and $\tau_{d}=8.45 \mathrm{~ms}$ for the average parameters determined from the fits.

\section{Subthreshold dynamics of the two-compartment model}

The impedance $Z(f)$ of the two-compartment model can be obtained from Equations 5 by neglecting the active spike-generating current and assuming a sinusoidal somatic current injection, or directly from the equivalent electrical model shown in Figure 1:

$$
Z(f)=\frac{g_{j}+g_{d}+2 \pi i C_{d} f}{\left(g_{j}+g_{d}+2 \pi i C_{s} f\right)\left(g_{j}+g_{d}+2 \pi i C_{d} f\right)-g_{j}^{2}} .
$$

As shown in Figure $4 B$, the amplitude of $Z$ decreases sharply before displaying an extended plateau up to very high frequencies. The origin and characteristics of this plateau can be understood by inspecting the circuit diagram in Figure 1. For frequencies larger than the inverse of the membrane time constant multiplied by $2 \pi, f>c_{m} /\left(2 \pi g_{m}\right)$, the membrane resistance is larger than the capacitive impedance in each compartment. The membrane resistance of each compartment is thus short-circuited by the compartment capacitance and can be neglected. In this regime, the two-compartment model equivalent electrical circuit reduces to a two-branch circuit with the somatic capacitance in one branch and the junctional conductance and the dendritic capacitance in series in the other:

$$
\frac{1}{Z(f)}=2 \pi i C_{s} f+\frac{1}{1 / g_{j}+1 /\left(2 \pi i C_{d} f\right)} .
$$

When $2 \pi f>C_{d} / g_{j} \simeq \tau_{d}$, the impedance of the second branch is dominated by the junctional conductance, and the contribution of the dendrite capacitance can be neglected. The impedance of this dendritic branch is also much smaller than the impedance of the somatic branch until one reaches frequencies such that $2 \pi f \simeq C_{s} / g_{j} \simeq \tau_{s}$, which are high enough for the capacitive somatic impedance to decrease to a value comparable with the junctional resistance. As a consequence, the impedance of the two-compartment model behaves purely resistively, $Z(f) \simeq g_{\text {; }}$, from frequencies of the order of $1 / 2 \pi \tau_{d}$, up to very high frequencies of the order of $1 / 2 \pi \tau_{s}$.

\section{Mathematical analysis of the firing modulation in the two-compartment model}

The firing modulation in the two-compartment model of the Purkinje cell can be understood with the help of a mathematical analysis. Here we give the details of this mathematical treatment, which relies on different approximations in different regime. The starting point are Equations $6-11$, which describe the dynamics of the somatic and dendritic compartments. For the parameter values determined from experiments, $g_{j} \gg$ $g_{s}$ and $g_{j} \gg g_{d}$, so that $\tau_{s} \sim c_{m} A_{s} / g_{j}, \tau_{d} \sim c_{m} A_{d} / g_{j}, g_{j, s} \sim 1$, and $g_{j, d} \sim 1$.

Input in the somatic compartment, noise in the dendritic compartment

We first consider the case in which the oscillatory input is injected in the soma, and the background noise is injected only in the dendritic compartment:

$$
\begin{gathered}
\tau_{s} \frac{d V_{s}}{d t}=-V_{s}+\Delta_{T} \exp \left(\frac{V-V_{T}}{\Delta_{T}}\right)+g_{j, s} V_{d}+\mu_{\text {inp }, s}(t) \\
\tau_{d} \frac{d V_{d}}{d t}=-V_{d}+g_{j, d} V_{s}+\sigma \sqrt{\tau_{d}} \eta
\end{gathered}
$$

where $\eta$ is a Gaussian process satisfying $\langle\eta\rangle=0$ and $\left\langle\eta(t) \eta\left(t^{\prime}\right)\right\rangle=\delta\left(t-t^{\prime}\right)$ and $\mu_{\text {inp }, \mathrm{s}}=\mu_{0}+\mu_{1} \sin (2 \pi f t)$.

Low-frequency approximation: $2 \pi \mathrm{f} \ll 1 / \tau_{\mathrm{s}}$. In the low-frequency regime, we neglect the exponential term, so that the model becomes equivalent to a two-compartment leaky integrate-and-fire model.

Because $\tau_{s} \ll \tau_{d}$, we eliminate the fast relaxing variable $V_{s}$ (see Fig. 7). As long as the input current to the soma varies on frequencies smaller than $2 \pi / \tau_{s}$, the membrane potential relaxes instantaneously to its stationary value:

$$
V_{s}(t)=g_{j, s} V_{d}+\mu_{0}+\mu_{1} \sin (2 \pi f t) .
$$

Inserting this value into Equation 18, we obtain an equivalent onecompartment model:

$$
\begin{aligned}
\tau_{m} \frac{d V_{d}}{d t}=-V_{d}+\frac{g_{j, d}}{1-g_{j, d} g_{j, s}}\left(\mu_{0}+\mu_{1} \sin (2 \pi f t)\right) & \\
& +\frac{\sigma}{1-g_{j, d} g_{j, s}} \sqrt{\tau_{d}} \eta(t),
\end{aligned}
$$

where the time constant is given by the following:

$$
\tau_{m}=\frac{C_{d}}{g_{d}+\frac{g_{s}}{1+g_{s} / g_{j}}} \approx \frac{c_{m}}{g_{m}}=200 \mathrm{~ms}
$$

The firing threshold for the equivalent single-compartment model is set by $V_{s}\left(V_{d}\right)=V_{\text {th }}$, where $V_{s}\left(V_{d}\right)$ is given by Equation 19 . The effective threshold for the equivalent model is thus given by the following: 


$$
\tilde{V}_{t h}=\frac{V_{t h}-\mu_{0}-\mu_{1} \sin (2 \pi f t)}{g_{j, s}} .
$$

When $V_{d}$ reaches $\tilde{V}_{\text {th }}$, its value is reset to $\tilde{V}_{r}=\tilde{V}_{\text {th }}-\beta$, where $\beta$ is the value of dendritic reset.

The effective threshold $\tilde{V}_{\text {th }}$ and reset $\tilde{V}_{r}$ both depend on the input current to the soma of the original two-compartment model and thus vary sinusoidally in time. To eliminate the dependence on time, it is useful to define a new variable $U$ as follows:

$$
U=g_{j, s} V_{d}+\mu_{1} \sin (2 \pi f t) .
$$

In terms of this new variable, the dynamics of the equivalent singlecompartment model become:

$$
\begin{aligned}
\tau_{m} \frac{d U}{d t}= & -U+\frac{g_{j, s} g_{j, d}}{1-g_{j, s} g_{j, d}} \mu_{0}+\frac{g_{j, s} g_{j, d}}{1-g_{j, s} g_{j, d}} \mu_{1} \sin (2 \pi f t) \\
& +\mu_{1} 2 \pi \tau_{m} f \sin \left(2 \pi f t+\frac{\pi}{2}\right)+\frac{g_{j, s}}{\sqrt{1-g_{j, s} g_{j, d}}} \sigma \sqrt{\tau_{m}} \eta(t),
\end{aligned}
$$

where the threshold value is $U_{\text {th }}=V_{\text {th }}$, and the reset value is $U_{r}=V_{\text {th }}-\beta g_{j s}$.

Note that, in this equivalent model, the sinusoidal modulation of the current has two components: one of amplitude proportional to $\mu_{1}$ and another of amplitude proportional to $f \mu_{1}$. Therefore, the firing-rate modulation $R_{2 C}(f)$ of the two-compartment model to a sinusoidal modulation in the soma is equal to the sum of single-compartment responses to the two components of the current:

$$
R_{2 c}(f)=\left(\frac{g_{j, s} g_{j, d}}{1-g_{j, s} g_{j, d}}+2 \pi \tau_{m} f e^{i \frac{\pi}{2}}\right) R_{1 c}(f) .
$$

The firing-rate modulation $R_{1 C}(f)$ in a single-compartment model is known analytically (Brunel and Hakim, 1999; Brunel et al., 2001). In particular, because the time constant $\tau_{m}$ is $200 \mathrm{~ms}$, the amplitude of $R_{1 C}(f)$ decreases as $f^{-1 / 2}$ for frequencies approximately above $\nu_{0}$; because of the current component of amplitude $\sim f \mu_{1}$ in the equivalent model, the amplitude of modulation in the two-compartment model increases as $f^{1 / 2}$ :

$$
R_{2 c}(f) \approx \frac{v_{0} \mu_{1}}{g_{j, s} \sigma} e^{i \frac{\pi}{4}} \sqrt{4 \pi f \tau_{d}}
$$

thus giving rise to the high-frequency amplification seen in the simulations (see Fig. 5A, left). The amplitude of the modulation increases for input frequencies up to $\sim 1 / 2 \pi \tau_{s}$, above which the mapping to the singlecompartment model becomes inaccurate because the timescale of the somatic compartment is comparable with the timescale of the oscillating input and Equation 19 breaks down.

High-frequency approximation: $2 \pi \mathrm{f} \gg 1 / \tau_{\mathrm{s}}$. In the high-frequency regimen, the membrane potential in the dendritic compartment does not change during the period of an oscillation. Therefore, the model becomes equivalent to a single-compartment EIF model, in which, for large $f$ (Fourcaud-Trocmé et al., 2003):

$$
R_{2 c}^{\mathrm{HF}}(f)=\frac{v_{0} \mu_{1}}{\Delta_{T} i 2 \pi f \tau_{s}} .
$$

Preferred frequency. The preferred frequency $f_{*}$ in the two-compartment model can be estimated by determining the value at which the lowfrequency approximation in Equation 26 and the high-frequency approximation Equation 27 intersect. This yields the following:

$$
f_{*}=\left(\frac{g_{j, s} \sigma}{4 \Delta_{T}}\right)^{2 / 3} \frac{1}{\pi \tau_{d}^{1 / 3} \tau_{s}^{2 / 3}} .
$$

Comparison with simulations. Although the analysis presented above captures qualitatively the resonance observed in the two-compartment model (see Fig. $5 A$ ), the quantitative match between the calculations and simulations depends on the accuracy of the underlying approximations.
In the low-frequency analysis, the exponential spike-generating current was replaced by a hard threshold. This approximation is valid if the activation range $\Delta_{T}$ of the spike-generating current is small compared with the typical variations in the membrane potential. In the equivalent single-compartment model, the scale of the variations is determined by the distance between the threshold and the dendritic reset, set by the parameter $\beta$. In the model used in Figure $5 A, \Delta_{T}=0.75 \mathrm{mV}$ and $\beta=0.5$ $\mathrm{mV}$, so that one cannot neglect the width of the AP. As a consequence, quantitatively, the analytic predictions deviate strongly from the simulations. If the size of the dendritic reset $\beta$ is increased artificially, the analytic predictions match closely the simulations (see Fig. $7 C, D$ ). Increasing $\beta$ while keeping other parameters constant leads to an increase of the resonant frequency but does not qualitatively affect the shape of the rate modulation.

\section{Input and noise in the dendritic compartment}

In the case in which both the oscillatory input and background noise reach the dendritic compartment only, the dynamics of the twocompartment model are given by the following:

$$
\begin{gathered}
\tau_{s} \frac{d V_{s}}{d t}=-V_{s}+\Delta_{T} \exp \left(\frac{V-V_{T}}{\Delta_{T}}\right)+g_{j, s} V_{d}, \\
\tau_{d} \frac{d V_{d}}{d t}=-V_{d}+g_{j, d} V_{s}+\mu_{\text {inp }, s}(t)+\sigma \sqrt{\tau_{d}} \eta,
\end{gathered}
$$

where $\eta$ is a Gaussian process satisfying $\langle\eta\rangle=0$ and $\left\langle\eta(t) \eta\left(t^{\prime}\right)\right\rangle=\delta\left(t-t^{\prime}\right)$ and $\mu_{\text {inp }}=\mu_{0}+\mu_{1} \sin (2 \pi f t)$.

The above analysis still holds, but Equation 19 becomes simply

$$
V_{s}(t)=g_{j, s} V_{d} .
$$

As a result, in the equivalent single-compartment model, the oscillatory input has a single component proportional to $\mu_{1}$. The oscillatory component with amplitude $f \mu_{1}$ that was present in the case of a somatic input is now absent, and, as a consequence, the modulation amplitude decreases monotonically with input frequency, as seen in Figure $5 B$. For intermediate frequencies, this decrease scales as $f^{-1 / 2}$, whereas for large frequencies, it follows $f^{-1}$.

Input and noise in the somatic compartment. In the case in which both the oscillatory input and background noise reach the somatic compartment only, the dynamics of the two-compartment model are given by the following:

$$
\tau_{s} \frac{d V_{s}}{d t}=-V_{s}+\Delta_{T} \exp \left(\frac{V-V_{T}}{\Delta_{T}}\right)+g_{j, s} V_{d}+\mu_{\text {inp }, s}(t)+\sigma \sqrt{\tau_{d}} \eta,
$$

$$
\tau_{d} \frac{d V_{d}}{d t}=-V_{d}+g_{j, d} V_{s}
$$

where $\eta$ is a Gaussian process satisfying $\langle\eta\rangle=0$ and $\left\langle\eta(t) \eta\left(t^{\prime}\right)\right\rangle=\delta\left(t-t^{\prime}\right)$ and $\mu_{\text {inp }, \mathrm{s}}=\mu_{0}+\mu_{1} \sin (2 \pi f t)$.

These dynamics are equivalent to a single-compartment EIF model with an additional membrane current (Brunel et al., 2003; Richardson et al., 2003). In this case, for strong background noise, the firing-rate modulation has a shape similar to the subthreshold impedance $Z(f)$ (Eq. 15).

As shown in Figure $4 B$ and discussed above, the amplitude of $Z$ decreases sharply up to a frequency of the order of $1 / 2 \pi \tau_{d}$ and then displays a plateau up to a frequency of the order of $1 / 2 \pi \tau_{s}$ before decaying to zero. The amplitude of the firing modulation in the two-compartment model with somatic input and noise displays a similar behavior (see Fig. $5 C$ ).

\section{Results}

Single-compartment and multicompartment models of the Purkinje cell predict qualitatively different responses to highfrequency inputs

We first set out to quantify computationally the firing-rate modulation of Purkinje cells using models of different complexities 
A

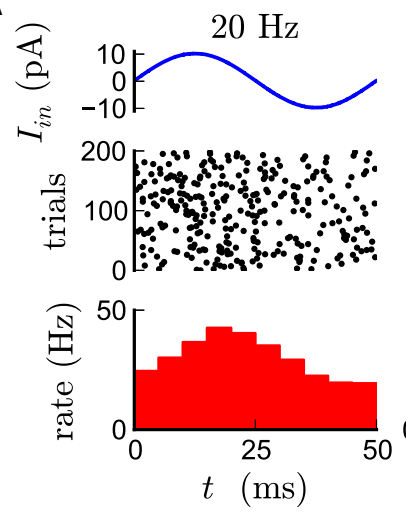

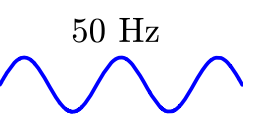
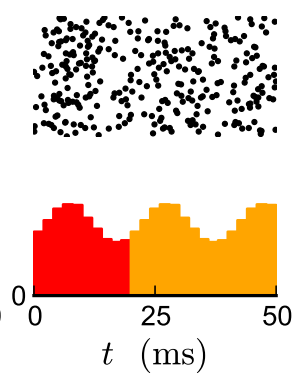

$t \quad(\mathrm{~ms})$

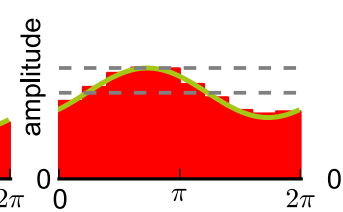

phase (rad)
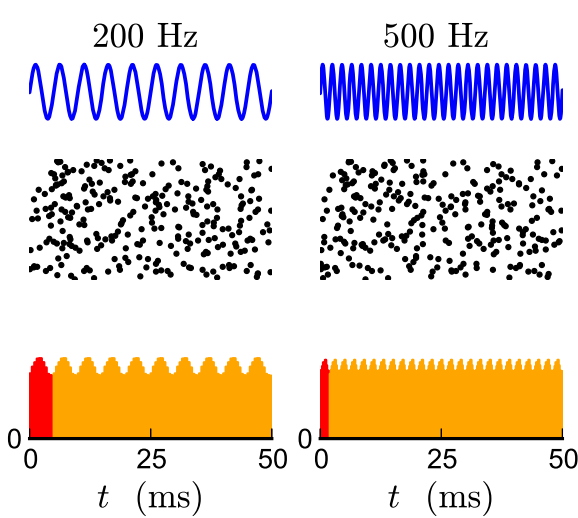

B

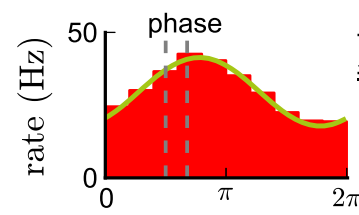

phase (rad) $t(\mathrm{~ms})$

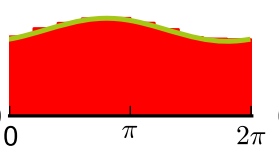

phase (rad)
$500 \mathrm{~Hz}$
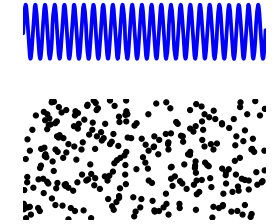

$t \quad(\mathrm{~ms})$

C
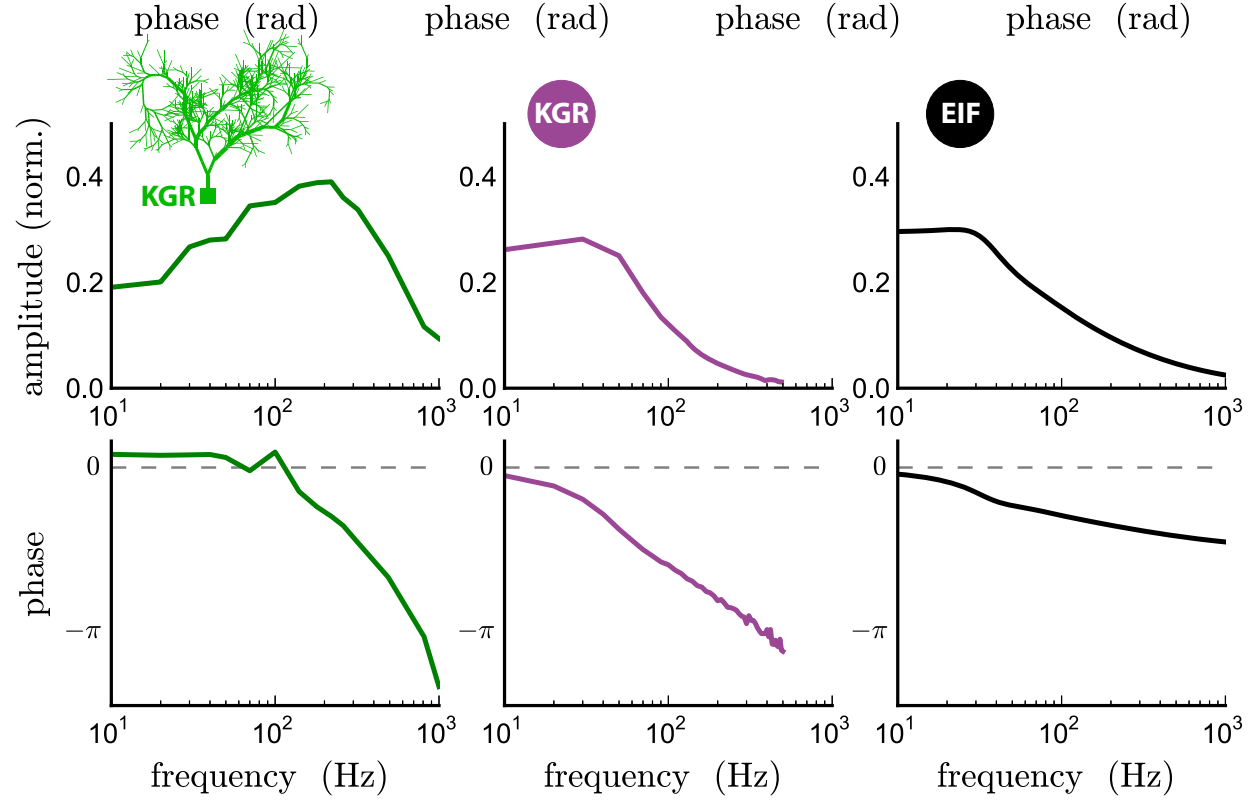

Figure 2. Firing modulation elicited by sinusoidal input currents in Purkinje cell models of different complexities. $A$, Illustration of the protocol for the measurement of the firing-rate modulation at a given frequency: a sinusoidal current of frequency $f$ (top row) is injected into a single-compartment model cell on top of a noisy background (data not shown); spike times are recorded over many repetitions of the input (middle row) and used to estimate the instantaneous firing rate (bottom row). $\boldsymbol{B}$, The modulation of the firing rate over one period of the input is well described by a sinusoidal variation around the mean firing rate at the frequency $f$ of the input; the amplitude and phase of the corresponding sinusoidal fit are used to quantify the modulation. The data shown in $A$ and $B$ was obtained from a single-compartment EIF model with a noisy background input leading to a mean firing rate of $30 \mathrm{~Hz}$ with a CV of 0.8 (membrane potential SD of $6 \mathrm{mV}$ ). C, Amplitude (normalized by the mean firing rate) and phase of the firing modulation as function of the input frequency, for three different models of the Purkinje cell: (1) a multicompartment conductance-based model (left); (2) a single-compartment conductance-based model (middle); and (3) a single-compartment ElF model (right). The mean firing rate was set to $30 \mathrm{~Hz}$ in the three models. Note that, here and in the following figures, we do not consider firing-rate modulations $<10 \mathrm{~Hz}$. This low-frequency range is known to be shaped by the specific conductances of the cell and is not the focus of the present work.

driven with oscillating input currents superposed on a noisy background input (Fig. $2 A, B$ ). We first examined a multicompartment model based on a freely available morphological reconstruction of a guinea pig Purkinje cell (Shelton, 1985; Miyasho et al., 2001). We implemented in the soma a biophysically detailed description of the active mechanisms of the Purkinje cell (Khaliq et al., 2003). In this model (KGR), AP generation relies on a specific, resurgent sodium current that induces spontaneous firing via a pacemaking mechanism (Raman and Bean, 2001; Khaliq et al., 2003; Akemann and Knöpfel, 2006). This model included seven active conductances implemented using NEURON (see
Materials and Methods). Hyperpolarization-activated $I_{h}$ channels were inserted in the dendrites, and a noisy background input was implemented using individual conductance-based synapses. The sinusoidal input current was injected in the somatic compartment.

The amplitude of this modulation was expected to decrease with the frequency of the oscillating input (Fig. $2 A, B$ ), as a basic consequence of the capacitive nature of the cell membrane. In strong contrast with this expectation, the firing-rate modulation was observed to increase with input current frequency up to $\sim 200 \mathrm{~Hz}$ and decayed for larger frequencies (Fig. 2C, left) but was 
A

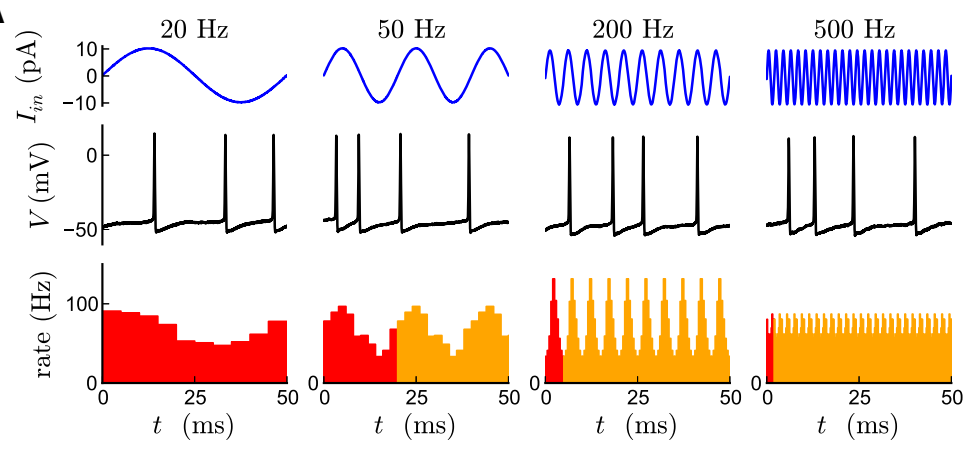

B

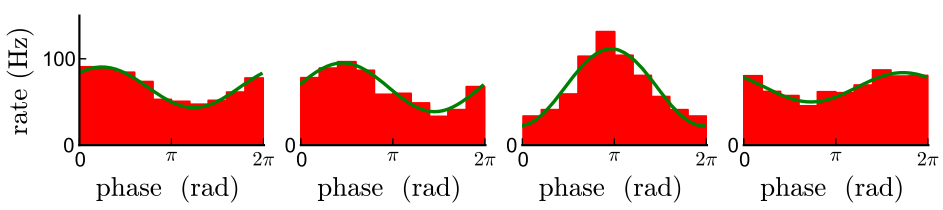

C
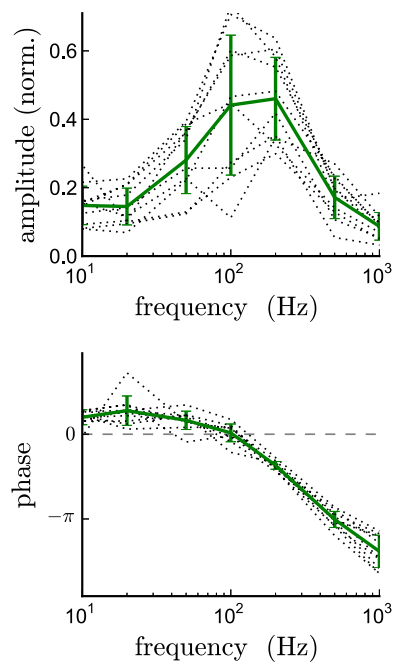

Figure 3. Boosting of high-frequency firing modulation in Purkinje cells in vitro. $\boldsymbol{A}$, Example of recorded responses in a typical cell. Top, Injected current waveforms; middle, modulation of the membrane potential in a single trial; bottom, instantaneous firing rate estimated by averaging over trials. $\boldsymbol{B}$, Sinusoidal fits to the single period average of the firing-rate modulation shown in $\boldsymbol{A}$. $\boldsymbol{C}$, Amplitude (normalized by the mean firing rate) and phase of the firing modulation as a function of the input frequency; mean \pm SD. Dashed lines, Individual cells ( $n=10$ cells from 4 animals); green lines, averages. The mean firing rate was $46 \pm 22 \mathrm{~Hz}$.

still approximately half the low-frequency modulation at $1 \mathrm{kHz}$. In addition, the phase of the modulation exhibited an advance up to $\sim 200 \mathrm{~Hz}$ instead of the expected lag.

To investigate the mechanisms of this amplification, we simulated a single-compartment model with the same KGR model of ionic channels as in the multicompartment model (Khaliq et al., 2003). As for the multicompartment model, the noisy background input was implemented using individual conductancebased synapses. In contrast to the multicompartmental model, numerical simulations showed low-pass behavior for the firingrate modulation by sinusoidal currents and no sign of highfrequency boosting (Fig. 2C, middle).

These results were very similar to those found for an EIF model (Fourcaud-Trocmé et al., 2003), which provides a simplified spike initiation mechanism based on a depolarizing current that depends exponentially and instantaneously on the voltage near threshold (see Materials and Methods). The EIF model has been shown to provide an effective description of AP initiation in Purkinje cells in the presence of noisy inputs (Rieubland et al., 2008). Despite faster AP initiation than in pyramidal cells (Badel et al., 2008), the Purkinje cell EIF model otherwise exhibited low-pass behavior as found in previous studies (Fourcaud-Trocmé et al., 2003; Richardson, 2007), with a low cutoff frequency, comparable with the mean firing rate (Fig. $2 C$, right). Moreover, in both single-compartment models, the phase of the modulation always lagged with respect to the input (negative phase in Fig. 2C, middle and right), in contrast to the advance seen in the multicompartment model up to $\sim 200 \mathrm{~Hz}$.

The low-pass behavior found in single-compartment models argued against active conductances being the basis of the amplification in the multicompartment model and motivated us to investigate further its underlying mechanism.

\section{In vitro measurements confirm boosting of spiking modulation at high frequencies}

Our numerical modeling predicted that firing modulation might be amplified in the Purkinje cell up to frequencies of several hundreds of Hertz. We tested this prediction using whole-cell patch-clamp current-clamp recordings in cerebellar sagittal slices prepared from adult rats and recorded under quasi-physiological conditions (potassium-based internal solution; $32^{\circ} \mathrm{C}$; see Materials and Methods).

We performed a linear analysis of the input-output transform of the Purkinje cell by injecting small sinusoidal currents $( \pm 10$ $\mathrm{pA})$ at defined frequencies $(2,5,10,20,50,100,200,500$, and $1000 \mathrm{~Hz}$ ) and noise (with an SD of $20 \mathrm{pA}$ ) while measuring the resulting modulation of the spontaneous spiking. The experimental approach is illustrated in Figure $3, A$ and $B$. Both the amplitude and the phase of the modulation displayed a strong similarity with the predictions of the multicompartment model (Fig. 2C, left): the amplitude increased up to a frequency of $\sim 200$ $\mathrm{Hz}$, and the phase exhibited a positive advance over the same frequency range. The resonance at $\sim 200 \mathrm{~Hz}$ means that highfrequency inputs have a particularly strong influence on the Purkinje cell output. We next set out to determine the mechanism of the boosting of high-frequency inputs.

\section{Subthreshold dynamics of the Purkinje cell show no sign of high-frequency amplification}

A possible mechanism that could explain the high-frequency amplification is an intrinsic resonance arising from the properties of voltage-dependent conductances. Subthreshold resonance has been observed in several cell types in the hippocampus and cortex (Gutfreund et al., 1995; Hutcheon et al., 1996; Leung and Yu, 1998; Pike et al., 2000; Hu et al., 2002, 2009; Narayanan and Johnston, 2008; Zemankovics et al., 2010) and can lead to firingrate resonance (Brunel et al., 2003; Richardson et al., 2003). However, resonant subthreshold frequencies reported so far have been confined to frequency ranges from a few Hertz to $\sim 50 \mathrm{~Hz}$, far below the resonant frequencies found in Purkinje cells (but see Remme et al., 2014). To investigate the possible existence of a subthreshold resonance, we injected a hyperpolarizing current to maintain the Purkinje cell below threshold and injected a "chirp," a sinusoidal current of continuously increasing frequency (Fig. $4 A$; see Materials and Methods). If there was a significant resonance around a particular frequency, the modulation of the 

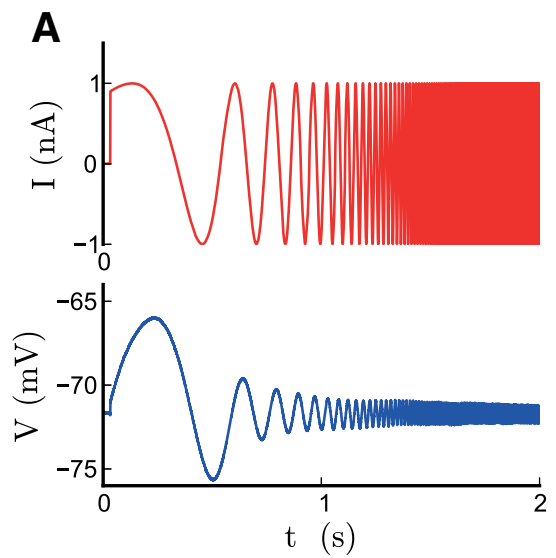

B
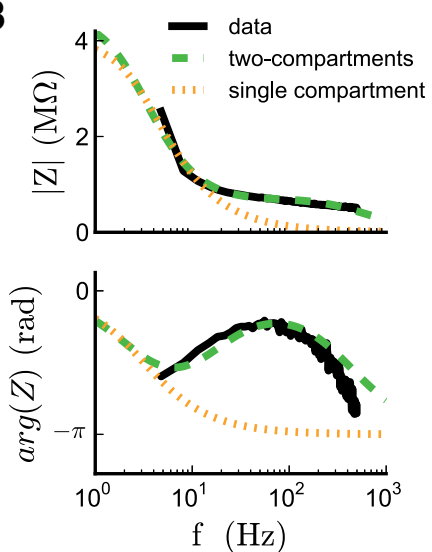
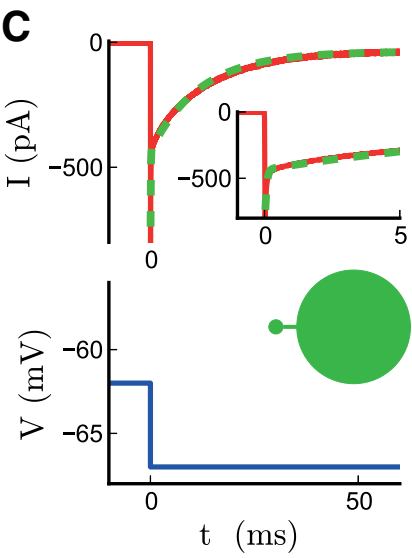

Figure 4. Subthreshold dynamics of the Purkinje cell. $\boldsymbol{A}$, Membrane potential dynamics (bottom) elicited by a current chirp (top). $\boldsymbol{B}$, Amplitude (top) and phase (bottom) of the complex impedance $Z$ of the cell determined from $\boldsymbol{A}$ as a function of input frequency compared with the impedances of single- and two-compartment models. $\boldsymbol{C}$, Current response (top) to a step of holding potential (bottom) in voltage clamp. Inset, Zoom on the first $5 \mathrm{~ms}$. The green trace is a fit by a two-compartment model symbolized here and subsequently by an asymmetric dumbbell (green).

membrane potential would be amplified at those frequencies. Figure $4 A$ shows the typical behavior of a Purkinje cell injected with a chirp current. There was no sign of any resonance, and the modulation of the membrane potential decreased monotonically ( $n=6$ cells from two animals). This is also seen in the subthreshold impedance of the cell (Fig. $4 B$ ), which shows separately the amplitude and phase of the membrane potential modulation as function of frequency.

Although the subthreshold impedance shows no resonance, it does display a remarkable phenomenon: the amplitude of the modulation decays very quickly at low frequencies but reaches a broad plateau extending from 50 to $500 \mathrm{~Hz}$. This behavior is at odds with the predictions of a passive, single-compartment model, in which the amplitude would decay steadily to zero (Fig. 4B).

Although the Purkinje cell is electrotonically highly compact (Shelton, 1985; Rapp et al., 1994), it is well known that the large membrane area of the dendrites has a great influence on transient currents (Llano et al., 1991; Roth and Häusser, 2001). Indeed, in contrast to a single-compartment model, the passive current response of a Purkinje cell to a voltage step exhibited at least two clearly separated timescales of relaxation (Fig. 4C): one in the submillisecond range and another of the order of tens of milliseconds. To quantify these timescales more precisely, we fitted the current transients of a two-compartment model to the experimental data (see Materials and Methods), which were recorded in the presence of $10 \mu \mathrm{M}$ ZD7288 (4-ethylphenylamino-1,2dimethyl-6-methylaminopyrimidinium chloride), which blocks $\mathrm{I}_{h}$. Although better fits could be obtained using a larger number of compartments (Roth and Häusser, 2001), a two-compartment model captured the two main timescales present in the current transient (Fig. 4C). The presence of an additional fast timescale compared with a single-compartment model allowed this purely passive two-compartment model to reproduce the highfrequency plateau seen in the subthreshold impedance (Fig. 4B).

The presence of two clearly distinct subthreshold timescales is a direct consequence of the morphology of the Purkinje cell. For all of the cells examined ( $n=5$ cells from two animals), the fits to two-compartment models found the area of the somatic compartment to be at least 50 times smaller than the area of the dendritic compartment, whereas the soma-to-dendrite junctional conductance was much larger than the membrane conductances of each compartment (see Materials and Methods). The capacitances of the two compartments were proportional to the compartment areas and therefore differed by a factor of 50 . This difference in the capacitances of the somatic and dendritic compartments results in two clearly separated capacitive timescales, the shorter one being submillisecond. We next show that the presence of these two timescales can account for the highfrequency amplification of firing modulation.

\section{A two-compartment model reveals the mechanism of morphology-induced resonance}

The numerical modeling reported above suggested that the morphology of the Purkinje cell needed to be taken into account to produce the high-frequency amplification seen in experiments, but which aspects of morphology are important still needed to be clarified. The analysis of subthreshold dynamics showed that morphology induces two clearly separated capacitive timescales, which can be captured by a passive two-compartment model. We now show that this minimal model, supplemented with a simple spike-generating mechanism, is sufficient to reproduce and explain the high-frequency amplification in the firing modulation.

We determined numerically the firing-rate modulation of a two-compartment EIF model in response to a sinusoidal current input in the somatic compartment, while both compartments received noisy background inputs. The amplitude of the modulation increased for input frequencies up to several hundreds of Hertz and decreased for higher frequencies (Fig. 5A), whereas the phase of the modulation displayed an advance at low frequencies and a lag at high input frequencies. These two features correspond closely to the experimental findings (Fig. 3C) and the predictions of the multicompartment model (Fig. 2C, left).

The two-compartment EIF model is sufficiently simple to allow for a detailed mathematical analysis of the dynamics (see Materials and Methods and Fig. 7). This analysis revealed the mechanism underlying high-frequency amplification. The first key element of the mechanism is the two-compartment nature of the Purkinje cell, which means that there is a broad range of frequencies at which the dendritic capacitance has an impedance that is negligible compared with the resistance linking soma and dendrites, whereas the impedance of the somatic capacitance is still much larger (Fig. 1; see Materials and Methods). At these frequencies, currents injected at the soma will experience an essentially resistive load $g_{j}$, and the amplitude of the voltage response to a sinusoidal current will be constant with frequency 
and in-phase (Fig. 4B), whereas it would decay with frequency as $1 / f$ in a single compartment.

The second key element of the mechanism is to recognize that the average firing rate of an ensemble of spontaneously active leakless neurons (with random phases) in the absence of noise is proportional to the time derivative of the membrane potential, as shown by Knight (1972). Intuitively, this arises simply from the fact that the larger $d V / d t$, the higher the rate of cells reaching threshold. Thus, if a modulation of voltage is introduced, the modulation of firing will be proportional to its derivative. For a sinusoidal voltage modulation, the firing-rate modulation will also be sinusoidal but with a phase advance of $\pi / 2$. This strictly holds only for leakless neurons, but it is true more generally for high-frequency modulation when conductance leakage is negligible. In the two-compartment model, the amplitude of the voltage modulation is essentially constant with frequency in the $50-500 \mathrm{~Hz}$ range, so the amplitude of the firing-rate modulation will increase linearly with frequency, because $d(\sin 2 \pi f t) / d t=$ $2 \pi f \cos 2 \pi f t$. This differs drastically from a one-compartment model, in which the linear increase with frequency is counterbalanced by the decay in the amplitude of the voltage response to the sinusoidal input.

These arguments qualitatively explain the amplification of high-frequency inputs and the phase advance in a range of frequencies. However, the amplification seen in Fig. 5 is sublinear, and the phase advance is smaller than $\pi / 2$. Furthermore, both amplitude and phase reach a maximum before decaying at very high frequencies. This is attributable to a combination of several effects. (1) It is known that the presence of white noise inputs decreases the amplitude of the firing-rate modulation by a factor $1 / \sqrt{\omega}$ and adds a phase shift of $\pi / 4$ compared with deterministic inputs (Brunel et al., 2001; for review, see Brunel and Hakim, 2008). (2) At a sufficiently high frequency, the somatic capacitance starts to play a role. It leads to a reduction of the ampli-

tude of the voltage modulation, and consequently of the amplitude of the firing-rate modulation, with frequency. (3) At very high frequencies, the dynamics of spike initiation leads to a $1 / f$ decay and a phase lag of $\pi / 2$ (Fourcaud-Trocmé et al., 2003; see Materials and Methods and Fig. 7).

The broad peak in the amplitude as a function of input frequency thus results from an interplay between the amplification at intermediate frequencies and the attenuation at very high frequencies. As a consequence, the preferred frequency is independent of the mean firing rate or its harmonics. Instead, the value of was $45 \mathrm{~Hz}$ in all three cases.
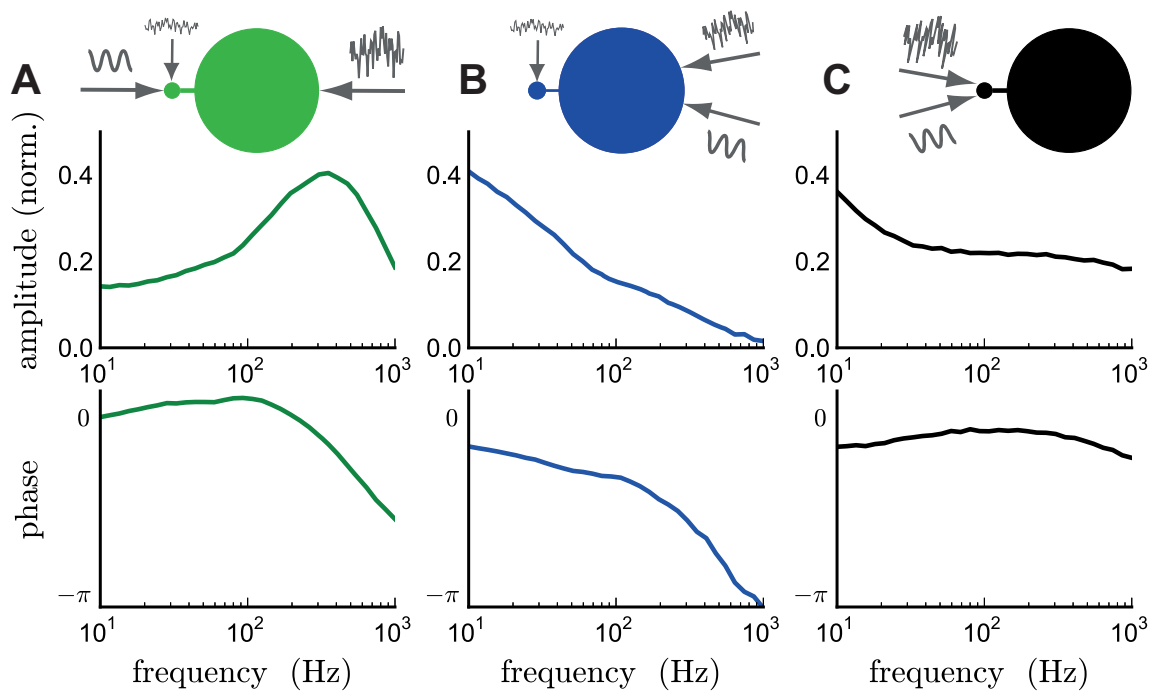

Figure 5. Firing modulation in the two-compartment EIF model. Amplitude (top) and phase (bottom) of the firing modulation as a function of the frequency of the input, elicited in three different conditions. $\boldsymbol{A}$, Input injected in the somatic compartment, background noise injected in both compartments. $\boldsymbol{B}$, Input injected in the dendritic compartment, background noise injected in both compartments. $\boldsymbol{C}$, Input injected in the somatic compartment, background noise injected in the somatic compartment only. The amplitude of the background noise was adjusted to produce the same interspike interval $C V$ as in $\boldsymbol{A}$ and $\boldsymbol{B}$. The mean firing rate
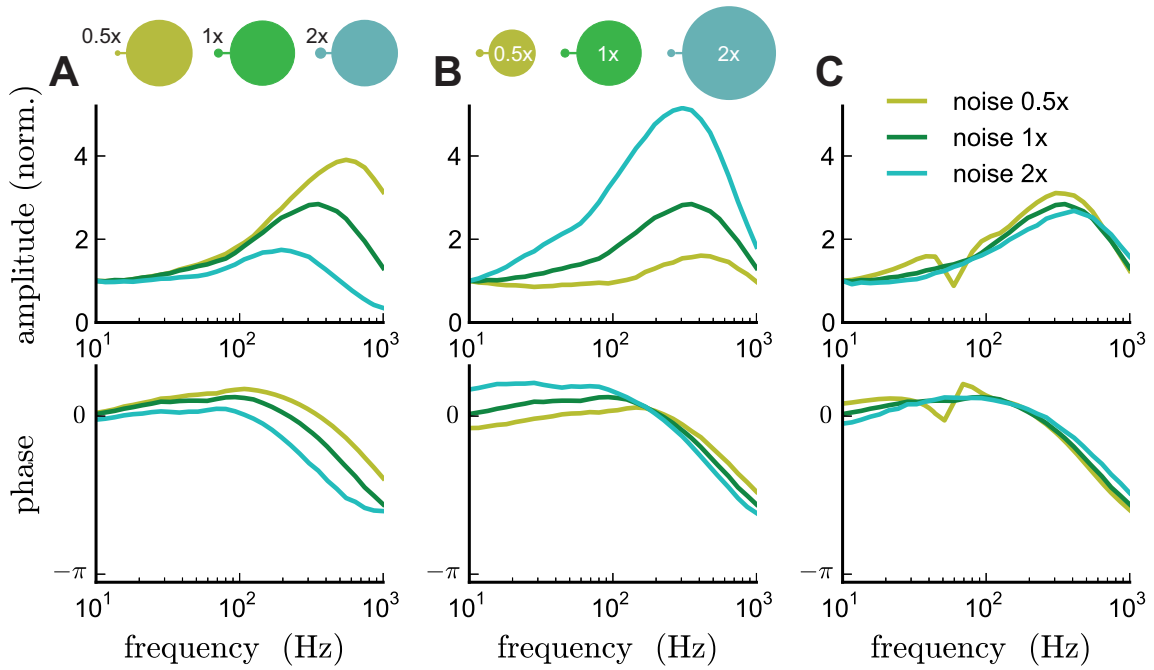

Figure 6. Morphology shapes the high-frequency resonance. $\boldsymbol{A}$, Influence of somatic compartment area on the firing modulation. The mean firing rates are 49,45 , and $35 \mathrm{~Hz}$ for increasing area. $\boldsymbol{B}$, Influence of dendritic compartment area on the firing modulation. The mean firing rate was $45 \mathrm{~Hz}$ in all three cases. $\boldsymbol{C}$, Influence of synaptic noise in the dendritic compartment area on the firing modulation. The mean firing rates are 55,45 , and $30 \mathrm{~Hz}$ for increasing noise. At low noise, resonances at multiples of the firing rate become visible. The location of these additional resonances is independent of the location of the main, high-frequency resonance. In each panel, a single parameter was increased/decreased by a factor of 2 , with all other parameter values kept as in Figure $5 \boldsymbol{A}$ ( $1 \times$ condition). For comparison, the firing modulation amplitude was normalized to the values at $1 \mathrm{~Hz}$. $\ln \boldsymbol{B}$, the mean and variance of the input were adjusted to keep the firing rate at $45 \mathrm{~Hz}$ and $\mathrm{CV}$ at 0.7 .

the preferred frequency depends on the relative size of the somatic and dendritic compartments (see Materials and Methods): smaller somatic compartments lead to stronger amplification and higher resonance frequencies (Fig. 6A); larger dendritic compartments induce a stronger amplification but at lower resonance frequencies (Fig. 6B). The precise location of the peak also appears to be affected by the strength of the post-spike afterhyperpolarization in the dendritic compartment (Fig. 7).

Therefore, the high-frequency amplification is mainly attributable to the fast subthreshold timescale induced by the Purkinje 

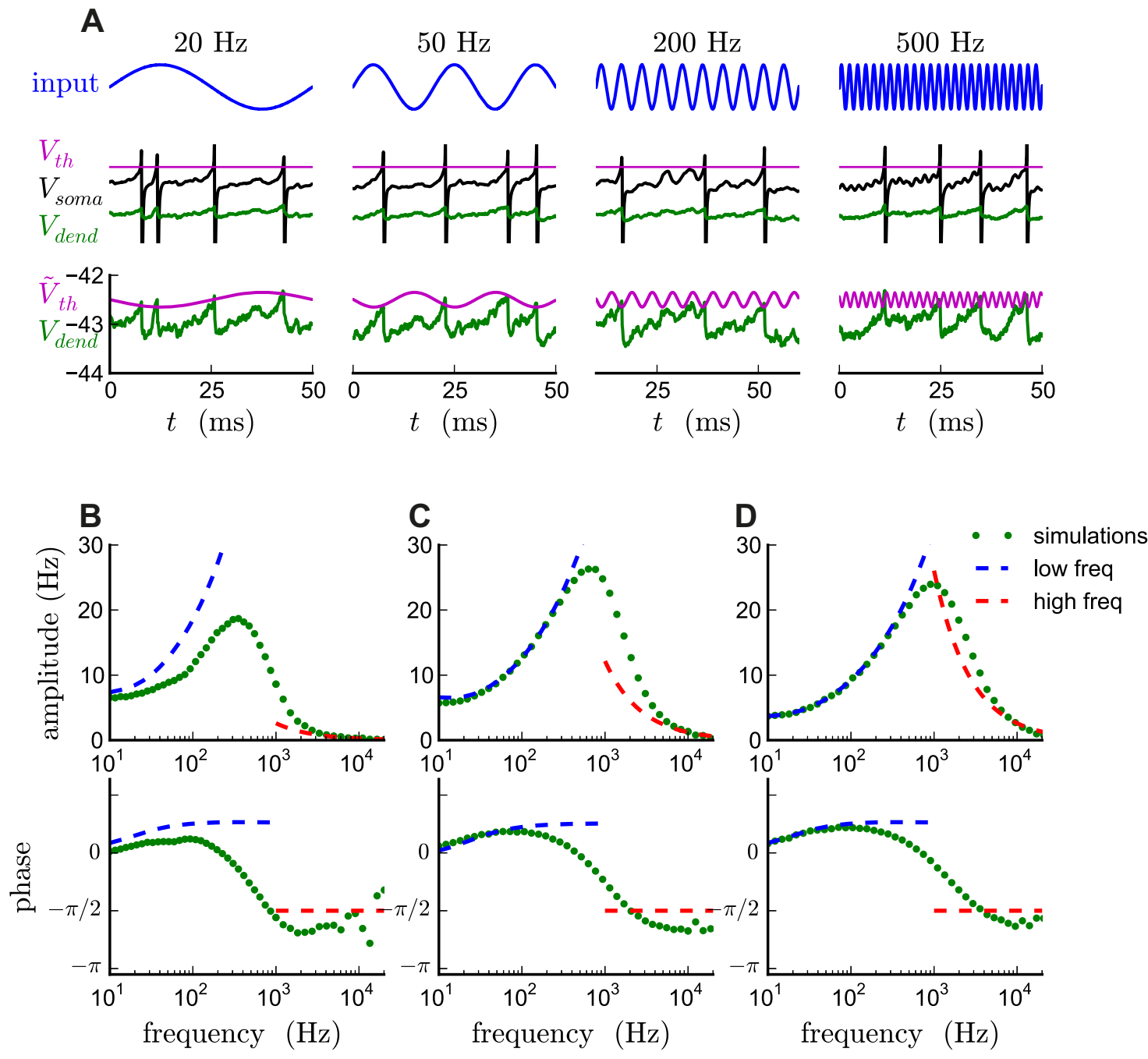

Figure 7. A, Illustration of the mapping from a two-compartment model to an effective single-compartment model. Top, Injected current; middle, membrane potential dynamics in the somatic and dendritic compartments (note the similarity in the fluctuations); bottom, membrane potential in the dendritic compartment compared with the effective, oscillating threshold. $\boldsymbol{B}$ - $\boldsymbol{D}$, Comparison between analytical results and simulations for three different values of the dendritic reset $\beta . \boldsymbol{B}, \beta=0.5 \mathrm{mV}$ as in Figure 5 . C, $\beta=3 \mathrm{mV}$. $\boldsymbol{D}, \beta=10 \mathrm{mV}$. All other parameters as in Figure 5. In $B$, the analytical curves were obtained by setting the mean input so as to reproduce the target firing rate of $45 \mathrm{~Hz}$ (the equivalent single-compartment model does not produce the correct current to rate relationship because $\beta$ is close to the AP onset range $\left.\Delta_{T}\right)$. In $($ and $D$, there are no free parameters in the analytical results.

cell morphology. However, the underlying mechanism relies on two additional ingredients. (1) The oscillating current input should be injected in the soma. If it is injected instead in the dendritic compartment, firing modulation is attenuated strongly with increasing frequency because of the low-pass filtering by the slow timescale of the dendritic compartment (Fig. 5B). (2) The bulk of the background synaptic noise should be injected in the dendritic compartment. If strong synaptic noise reaches only the somatic compartment, the firing modulation resembles the subthreshold impedance (Brunel et al., 2003; Richardson et al., 2003): it still displays a pronounced plateau at high frequencies, but no resonance is present (Fig. 5C). In contrast, increasing the strength of synaptic fluctuations in the dendritic compartment affects only weakly the high-frequency resonance (Fig. 6C).

Purkinje cell morphology and an artificial threshold are sufficient to reproduce the high-frequency resonance

Our modeling analysis suggests that the resonance can arise from the interaction of the Purkinje cell morphology and a firing mechanism represented by the simplest possible threshold. We sought to test this directly in a series of experiments designed to demonstrate resonance in Purkinje cells with an artificial threshold. To do this, we blocked voltage-dependent sodium and potassium channels by including $5 \mathrm{~mm}$ QX-314 and $5 \mathrm{~mm}$ TEA in the intracellular solution (see Materials and Methods) and introduced an electronic threshold and reset that approximated a simple formal firing mechanism. The persistence of resonance under such conditions would show that it does not depend on details of the firing mechanism and therefore only on the passive properties of the cell.

The operation of the circuit is explained in the diagram of Figure $8 A$. A dual recording was established to inject currents via one electrode without interfering with voltage recording via the second. The voltage recording was connected to a comparator that implemented a "hard" threshold. Whenever the membrane potential exceeded a user-set value (approximately $-80 \mathrm{mV}$ for the cell illustrated; potentials negative to those of normal firing were always set), a pulse was triggered. The comparator operated essentially instantaneously compared with the timescale of cellular behavior; therefore, response time was only limited by the filtering inherent in the patch-clamp recordings. The pulse 


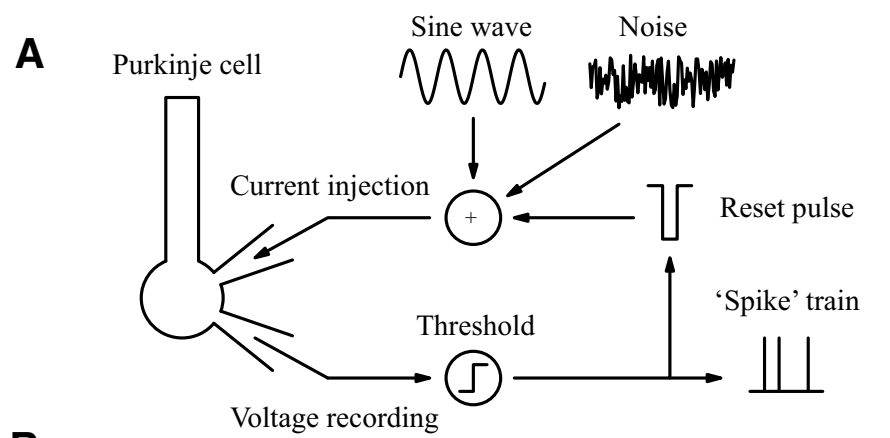

B

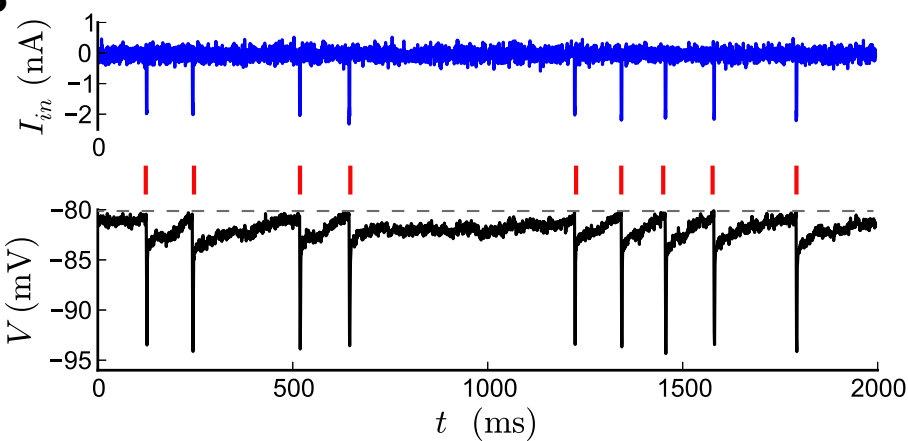

C
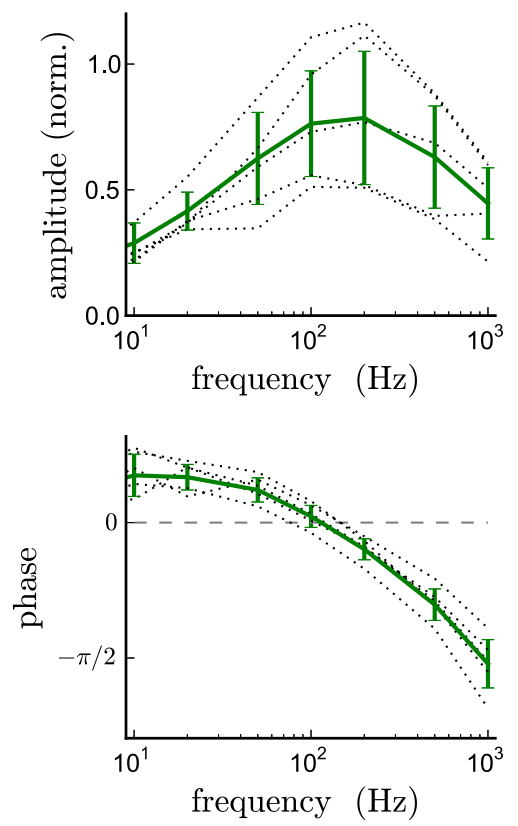

Figure 8. High-frequency resonance in a Purkinje cell with an artificial threshold. $\boldsymbol{A}$, Diagram illustrating the artificial firing mechanism used to evaluate Purkinje cell firing in the absence of native voltage-dependent conductances. They were blocked, and an electronic threshold was imposed on the recorded membrane potential. Whenever the threshold was crossed, the membrane potential was reset by a hyperpolarizing current pulse summed with the ongoing sine wave and noise stimuli. $\boldsymbol{B}$, Example of injected current (top) and the resulting membrane potential dynamics (bottom). APs (in red) occur at times when the membrane potential crosses the threshold value (dashed line). $C$, Amplitude (normalized by the mean firing rate) and phase of the firing modulation as a function of the input frequency; mean \pm SD. Dashed lines, Individual cells ( $n=6$ cells); green lines, averages. The mean firing rate was $12 \pm 2 \mathrm{~Hz}$.

served two purposes: it indicated the emission of an "action potential" and also caused a brief current pulse to be added to the current command. This hyperpolarized the membrane potential and therefore reset it below the threshold. The pulse was approximately $-2 \mathrm{nA}$ and lasted for $\sim 2 \mathrm{~ms}$. Figure $8 B$ illustrates the input current, the membrane potential, and "spike" train during these experiments.

As in the experiments above, we injected a series of sinusoidal currents at different frequencies summed with noise (SD of $60 \mathrm{pA}$ ) and the reset pulses. The holding current required occasional adjustment to maintain a relatively low firing frequency $(<20 \mathrm{~Hz})$, and analysis was restricted to $5 \mathrm{~s}$ sweeps that satisfied this criterion to prevent confusion between harmonics of the firing frequency and the highfrequency resonance for which we were testing. The recordings were performed in the presence of $100 \mu \mathrm{M}$ ZD7288, an inhibitor of $I_{h}$, and $5 \mu \mathrm{M}$ gabazine.

As can be seen in Figure $8 C$, the firing modulation increased clearly with frequency, and a high-frequency resonance was apparent. In all $n=6$ cells recorded, the amplitude of the response modulation was greater at 100 and $200 \mathrm{~Hz}$ than at $1 \mathrm{~Hz}$. The ratio between the amplitudes at 100 and $1 \mathrm{~Hz}$ was $4.1 \pm 1.3$ (mean \pm SEM), whereas the ratio between the amplitudes at 200 and $1 \mathrm{~Hz}$ was $4.2 \pm 0.7$. Testing the differences between the modulation amplitudes at 100 and $1 \mathrm{~Hz}$ with the Wilcoxon's rank-sum test yielded $p=0.002$. The same $p$ value was obtained for the differences between the amplitudes at 200 and $1 \mathrm{~Hz}$. This confirms that signal amplification at high frequencies is independent of the kinetic properties of the channels implementing the AP and only requires the operation of the simplest threshold in the context of the passive electrical properties of the cells, which are primarily determined by its morphology.

\section{Discussion}

We have identified a novel mechanism, morphology-induced resonance, that can amplify neuronal responses to highfrequency somatic inputs and counteract the attenuating effect of the membrane capacitance at high input frequencies. The extension of bandwidth of the firing modulation response is quite dramatic-approximately two decades of frequency in the Purkinje cell-compared with the equivalent single-compartment model. Indeed, the amplification mechanism is sufficiently powerful to create high-frequency resonance.

In contrast to the extensively studied phenomenon of subthreshold resonance (Hutcheon and Yarom, 2000), which is generally limited to lower frequencies (but see Remme et al., 2014), one surprising aspect of morphology-induced resonance is that it requires no specific voltage-dependent conductances active at subthreshold voltages. Only voltage-dependent conductances with very rapid kinetics and active during initiation of the AP could strongly influence the resonance. Thus, our modeling demonstrated that the simplest possible spiking models were sufficient, when combined with the two-compartment morphology in the presence of dendritic noise, to amplify high-frequency inputs.

We confirmed the existence of the predicted high-frequency amplification with recordings in cerebellar Purkinje cells, in which the somatodendritic dichotomy is especially marked (Llano et al., 1991). In good agreement with the modeling predictions, the rate of tonic firing of Purkinje cells could be modulated by somatic injections of sinusoidal currents up to remarkably high frequencies $(\sim 1 \mathrm{kHz})$. This implies that Purkinje cells are able to react on a submillisecond timescale to such inputs. Furthermore, Purkinje cells were shown to amplify oscillatory input through a broad resonance at frequencies at $\sim 200 \mathrm{~Hz}$. 
The firing-rate response of Purkinje cells characterized here can be compared with similar measurements in cortical pyramidal cells (Köndgen et al., 2008; Boucsein et al., 2009; Tchumatchenko et al., 2011; Ilin et al., 2013; Testa-Silva et al., 2014). In those studies, cortical pyramidal cells were shown to exhibit flat responses with cutoff frequencies of the order of several hundred Hertz. These cutoff frequencies are much higher than those obtained in standard Hodgkin-Huxley-type models (FourcaudTrocmé et al., 2003; Huang et al., 2012; Ilin et al., 2013), unless sodium channels exhibit cooperative gating (Huang et al., 2012; Ilin et al., 2013). The mechanism we propose offers an additional insight into recent modeling observations by Eyal et al. (2014), who argued that the extended bandwidth of pyramidal cell firing is controlled by dendritic impedance through alteration of spike initiation. The absence of attenuation over most of the frequency range is in itself a surprising finding. Our analysis suggests that it results from the action of the somatodendritic asymmetry independently of the details of the spiking mechanism. However, the cutoff frequency of this extended response range is determined by the details of spike initiation. Moreover, we show that, if dendritic noise dominates over somatic noise, the firing modulation is amplified strongly for frequencies up to several hundreds of Hertz, leading to high-frequency resonance. Such a highfrequency resonance has not been found so far in pyramidal cells (but see Higgs and Spain, 2009). This might be attributable to several factors: differences in morphology (Fig. 6) or strong noise injected at the soma (Fig. $5 C$ ).

The extension of bandwidth and resonance provided by morphology-induced resonance is predicted by our modeling study to operate exclusively on somatic inputs, like those used to confirm the existence of the phenomenon experimentally. This specificity was not recognized previously and represents a fundamental asymmetry between excitatory inputs, which in pyramidal and Purkinje cells are uniformly dendritic, and inhibitory inputs, of which a part targets the soma. The consequence of this is that these cells can respond extremely rapidly to fast somatic inhibitory inputs, which are moreover subject to resonant amplification. Therefore, morphology-induced resonance is expected to enhance the speed and efficacy of all of the functions that are attributed to somatic inhibition, including in particular feedforward inhibition (Brunel et al., 2004; Mittmann et al., 2005). Although the fastest inhibition in Purkinje cells is supplied via ephaptic transmission at the pinceau (Blot and Barbour, 2014) rather than chemical synaptic inhibition, the latter plays an essential role, rapidly "taking over" from the transient ephaptic inhibition.

More specifically, somatic inputs are widely believed to play key roles in the genesis of high-frequency oscillations in the gamma band and/or $\sim 200 \mathrm{~Hz}$ in several brain regions. Morphology-induced resonance can facilitate and select these oscillations. Furthermore, this resonance will be enhanced by dendritic noise and attenuated by somatic noise, offering hitherto unexplored mechanisms of regulation. In the cerebellum, a 200 $\mathrm{Hz}$ fast global oscillation (Brunel and Hakim, 1999) organizes the activity of the Purkinje cell population (Adrian, 1935; de Solages et al., 2008). The oscillation is thought to rely on fast mutual somatic inhibition arising from Purkinje cell axon collaterals, which provide oscillatory inputs to the soma of Purkinje cells (but see Watt et al., 2009). The morphology-induced resonance in Purkinje cells is almost perfectly matched with the frequency of their population oscillations, suggesting that the resonance is indeed a key mechanism in the emergence of the high-frequency oscillation. Thus, in addition to network mechanisms (Ledoux and Brunel, 2011) and subthreshold resonance caused by intrinsic conductances (Pike et al., 2000; Hu et al., 2002; Stark et al., 2013), morphology-induced resonance should be considered a novel source of neuronal and network resonance in the genesis of oscillations.

\section{References}

Adrian ED (1935) Discharge frequencies in the cerebral and cerebellar cortex. Proc Phys Soc 83:32-33.

Akemann W, Knöpfel T (2006) Interaction of Kv3 potassium channels and resurgent sodium current influences the rate of spontaneous firing of Purkinje neurons. J Neurosci 26:4602-4612. CrossRef Medline

Badel L, Lefort S, Brette R, Petersen CCH, Gerstner W, Richardson MJE (2008) Dynamic I-V curves are reliable predictors of naturalistic pyramidal-neuron voltage traces. J Neurophysiol 99:656-666. CrossRef Medline

Blot A, Barbour B (2014) Ultra-rapid axon-axon ephaptic inhibition of cerebellar Purkinje cells by the pinceau. Nat Neurosci 17:289-295. CrossRef Medline

Boucsein C, Tetzlaff T, Meier R, Aertsen A, Naundorf B (2009) Dynamical response properties of neocortical neuron ensembles: multiplicative versus additive noise. J Neurosci 29:1006-1010. CrossRef Medline

Broicher T, Malerba P, Dorval AD, Borisyuk A, Fernandez FR, White JA (2012) Spike phase locking in CAl pyramidal neurons depends on background conductance and firing rate. J Neurosci 32:14374-14388. CrossRef Medline

Brunel N, Hakim V (1999) Fast global oscillations in networks of integrateand-fire neurons with low firing rates. Neural Comput 11:1621-1671. CrossRef Medline

Brunel N, Hakim V (2008) Sparsely synchronized neuronal oscillations. Chaos 18:015113. CrossRef Medline

Brunel N, Chance FS, Fourcaud N, Abbott LF (2001) Effects of synaptic noise and filtering on the frequency response of spiking neurons. Phys Rev Lett 86:2186-2189. CrossRef Medline

Brunel N, Hakim V, Richardson M (2003) Firing-rate resonance in a generalized integrate-and-fire neuron with subthreshold resonance. Phys Rev E Stat Nonlin Soft Matter Phys 67:051916. CrossRef Medline

Brunel N, Hakim V, Isope P, Nadal JP, Barbour B (2004) Optimal information storage and the distribution of synaptic weights: perceptron versus Purkinje cell. Neuron 43:745-757. CrossRef Medline

de Solages C, Szapiro G, Brunel N, Hakim V, Isope P, Buisseret P, Rousseau C, Barbour B, Léna C (2008) High-frequency organization and synchrony of activity in the Purkinje cell layer of the cerebellum. Neuron 58:775-788. CrossRef Medline

Eyal G, Mansvelder HD, de Kock CP, Segev I (2014) Dendrites impact the encoding capabilities of the axon. J Neurosci 34:8063-8071. CrossRef Medline

Fourcaud-Trocmé N, Hansel D, van Vreeswijk C, Brunel N (2003) How spike generation mechanisms determine the neuronal response to fluctuating inputs. J Neurosci 23:11628-11640. Medline

Fuhrmann G, Markram H, Tsodyks M (2002) Spike frequency adaptation and neocortical rhythms. J Neurophysiol 88:761-770. Medline

Gutfreund Y, Yarom Y, Segev I (1995) Subthreshold oscillations and resonant frequency in guinea-pig cortical neurons: physiology and modelling. J Physiol 483:621-640. CrossRef Medline

Häusser M, Major G, Stuart GJ (2001) Differential shunting of EPSPs by action potentials. Science 291:138-141. CrossRef Medline

Higgs MH, Spain WJ (2009) Conditional bursting enhances resonant firing in neocortical layer 2-3 pyramidal neurons. J Neurosci 29:1285-1299. CrossRef Medline

Hu H, Vervaeke K, Storm JF (2002) Two forms of electrical resonance at theta frequencies, generated by $\mathrm{M}$-current, h-current and persistent $\mathrm{Na}+$ current in rat hippocampal pyramidal cells. J Physiol 545:783-805. CrossRef Medline

Hu H, Vervaeke K, Graham LJ, Storm JF (2009) Complementary theta resonance filtering by two spatially segregated mechanisms in CA1 hippocampal pyramidal neurons. J Neurosci 29:14472-14483. CrossRef Medline

Huang M, Volgushev M, Wolf F (2012) A small fraction of strongly cooperative sodium channels boosts neuronal encoding of high frequencies. PLoS One 7:e37629. CrossRef Medline

Hutcheon B, Yarom Y (2000) Resonance, oscillation and the intrinsic fre- 
quency preferences of neurons. Trends Neurosci 23:216-222. CrossRef Medline

Hutcheon B, Miura RM, Puil E (1996) Subthreshold membrane resonance in neocortical neurons. J Neurophysiol 76:683-697. Medline

Ilin V, Malyshev A, Wolf F, Volgushev M (2013) Fast computations in cortical ensembles require rapid initiation of action potentials. J Neurosci 33:2281-2292. CrossRef Medline

Isope P, Barbour B (2002) Properties of unitary granule cell-Purkinje cell synapses in adult rat cerebellar slices. J Neurosci 22:9668-9678. Medline

Khaliq ZM, Gouwens NW, Raman IM (2003) The contribution of resurgent sodium current to high-frequency firing in Purkinje neurons: an experimental and modeling study. J Neurosci 23:4899-4912. Medline

Knight BW (1972) Dynamics of encoding in a population of neurons. J Gen Physiol 59:734-766. CrossRef Medline

Köndgen H, Geisler C, Fusi S, Wang XJ, Lüscher HR, Giugliano M (2008) The dynamical response properties of neocortical neurons to temporally modulated noisy inputs in vitro. Cereb Cortex 18:2086-2097. Medline

Ledoux E, Brunel N (2011) Dynamics of networks of excitatory and inhibitory neurons in response to time-dependent inputs. Front Comput Neurosci 5:25. CrossRef Medline

Leung LS, Yu HW (1998) Theta-frequency resonance in hippocampal CA1 neurons in vitro demonstrated by sinusoidal current injection. J Neurophysiol 79:1592-1596. Medline

Lindner B, Schimansky-Geier L (2001) Transmission of noise coded versus additive signals through a neuronal ensemble. Phys Rev Lett 86:29342937. CrossRef Medline

Liu H, Agam Y, Madsen JR, Kreiman G (2009) Timing, timing, timing: fast decoding of object information from intracranial field potentials in human visual cortex. Neuron 62:281-290. CrossRef Medline

Llano I, Marty A, Armstrong CM, Konnerth A (1991) Synaptic- and agonist-induced excitatory currents of Purkinje cells in rat cerebellar slices. J Physiol 434:183-213. CrossRef Medline

Mittmann W, Koch U, Häusser M (2005) Feed-forward inhibition shapes the spike output of cerebellar Purkinje cells. J Physiol 563:369-378. CrossRef Medline

Miyasho T, Takagi H, Suzuki H, Watanabe S, Inoue M, Kudo Y, Miyakawa H (2001) Low-threshold potassium channels and a low-threshold calcium channel regulate $\mathrm{Ca} 2+$ spike firing in the dendrites of cerebellar Purkinje neurons: a modeling study. Brain Res 891:106-115. CrossRef Medline

Narayanan R, Johnston D (2008) The H channel mediates location dependence and plasticity of intrinsic phase response in rat hippocampal neurons. J Neurosci 28:5846-5860. CrossRef Medline

Ostojic S, Brunel N (2011) From spiking neuron models to linear-nonlinear models. PLoS Comput Biol 7:e1001056. CrossRef Medline

Ostojic S, Brunel N, Hakim V (2009) How connectivity, background activity, and synaptic properties shape the cross-correlation between spike trains. J Neurosci 29:10234-10253. CrossRef Medline

Pike FG, Goddard RS, Suckling JM, Ganter P, Kasthuri N, Paulsen O (2000) Distinct frequency preferences of different types of rat hippocampal neurones in response to oscillatory input currents. J Physiol 529:205-213. CrossRef Medline

Pinsky PF, Rinzel J (1994) Intrinsic and network rhythmogenesis in a re- duced Traub model for CA3 neurons. J Comput Neurosci 1:39-60. CrossRef Medline

Raman IM, Bean BP (2001) Inactivation and recovery of sodium currents in cerebellar Purkinje neurons: evidence for two mechanisms. Biophys J 80:729-737. CrossRef Medline

Rapp M, Segev I, Yarom Y (1994) Physiology, morphology and detailed passive models of guinea-pig cerebellar Purkinje cells. J Physiol 474:101118. CrossRef Medline

Remme MW, Donato R, Mikiel-Hunter J, Ballestero JA, Foster S, Rinzel J, McAlpine D (2014) Subthreshold resonance properties contribute to the efficient coding of auditory spatial cues. Proc Natl Acad Sci U S A 111:E2339-E2348. CrossRef Medline

Richardson M (2007) Firing-rate response of linear and nonlinear integrateand-fire neurons to modulated current-based and conductance-based synaptic drive. Phys Rev E Stat Nonlin Soft Matter Phys 76:021919. CrossRef Medline

Richardson MJE, Brunel N, Hakim V (2003) From subthreshold to firingrate resonance. J Neurophysiol 89:2538-2554. CrossRef Medline

Rieubland S, Davie JT, Roth A, Hausser M (2008) The dynamic currentvoltage relation of cerebellar Purkinje cells. Soc Neurosci Abs 34:44.16.

Roth A, Häusser M (2001) Compartmental models of rat cerebellar Purkinje cells based on simultaneous somatic and dendritic patch-clamp recordings. J Physiol 535:445-472. CrossRef Medline

Rousselet GA, Fabre-Thorpe M, Thorpe SJ (2002) Parallel processing in high-level categorization of natural images. Nat Neurosci 5:629-630. CrossRef Medline

Shelton DP (1985) Membrane resistivity estimated for the Purkinje neuron by means of a passive computer model. Neuroscience 14:111-131. CrossRef Medline

Stark E, Eichler R, Roux L, Fujisawa S, Rotstein HG, Buzsáki G (2013) Inhibition-induced theta resonance in cortical circuits. Neuron 80:12631276. CrossRef Medline

Tchumatchenko T, Malyshev A, Wolf F, Volgushev M (2011) Ultrafast population encoding by cortical neurons. J Neurosci 31:12171-12179. CrossRef Medline

Testa-Silva G, Verhoog MB, Linaro D, de Kock CP, Baayen JC, Meredith RM, De Zeeuw CI, Giugliano M, Mansvelder HD (2014) High bandwidth synaptic communication and frequency tracking in human neocortex. PLoS Biol 12:e1002007. CrossRef Medline

Tsodyks C, Sejnowski T (1995) Chaotic balanced state in a model of cortical circuits. Network 6:1-14. CrossRef

van Vreeswijk C, Sompolinsky H (1998) Chaotic balanced state in a model of cortical circuits. Neural Comput 10:1321-1371. CrossRef Medline

Watt AJ, Cuntz H, Mori M, Nusser Z, Sjöström PJ, Häusser M (2009) Traveling waves in developing cerebellar cortex mediated by asymmetrical purkinje cell connectivity. Nat Neurosci 12:463-473. CrossRef Medline

Womack M, Khodakhah K (2002) Active contribution of dendrites to the tonic and trimodal patterns of activity in cerebellar Purkinje neurons. J Neurosci 22:10603-10612. Medline

Zemankovics R, Káli S, Paulsen O, Freund TF, Hájos N (2010) Differences in subthreshold resonance of hippocampal pyramidal cells and interneurons: the role of h-current and passive membrane characteristics. J Physiol 588:2109-2132. CrossRef Medline 